\title{
Abnormal Regulation of High Affinity Nicotinic Receptors in Subjects with Schizophrenia
}

Charles R. Breese, Ph.D., Michael J. Lee, B.S., Cathy E. Adams, Ph.D., Bernadette Sullivan, B.S., Judy Logel, M.S., Kieron M. Gillen, B.S., Michael J. Marks, Ph.D., Allan C. Collins, Ph.D., and Sherry Leonard, Ph.D.

Previous studies have suggested that an abnormality in neuronal nicotinic acetylcholine receptor expression or function may be involved in the neuropathophysiology of schizophrenia. $\left[{ }^{3} \mathrm{H}\right]$-nicotine and $\left[{ }^{3} \mathrm{H}\right]$-epibatidine binding were compared in postmortem brain from control and schizophrenic subjects with varying smoking histories. In control subjects, increased receptor binding was seen in hippocampus, cortex, and caudate with increasing tobacco use. In contrast, schizophrenic smokers had reduced nicotinic receptor levels in these brain regions compared to control smokers. Chronic haloperidol and nicotine treatment, in the rat, was used to assess neuroleptic effects on receptor up-regulation by nicotine. A significant increase in cortical nicotinic receptors was seen in both nicotine treated as well as haloperidol and nicotine co-treated animals, suggesting that the abnormal regulation of high affinity neuronal nicotinic receptors in schizophrenics following nicotine use was not related to chronic neuroleptic treatment.

[Neuropsychopharmacology 23:351-364, 2000] (C) 2000 American College of Neuropsychopharmacology. Published by Elsevier Science Inc.
KEY WORDS: Schizophrenia; Cigarettes; Human brain; Nicotine; Nicotinic receptors; Postmortem; Receptor binding; Smoking

Previous studies have shown that high affinity nicotinic receptor numbers increase in the brains of human subjects who smoke (Benwell et al. 1988; Breese et al. 1997a; Court et al. 1998). This increase was shown to be dosedependent, based on the number of cigarettes smoked

From the Department of Psychiatry (CRB, CEA, BS, KMG, SL) and the Neurosciences Program (MJL, SL), University of Colorado Health Sciences Center, Denver, CO; Denver Veterans Administration Medical Center (JL, SL), Denver, CO; Institute for Behavioral Genetics, Department of Psychology (MJM, ACC), University of Colorado, Boulder, CO; and the Department of Pharmacal Sciences (CRB), Auburn University School of Pharmacy, Auburn, AL.

Address correspondence to: Dr. Sherry Leonard, Department of Psychiatry, Box C268-71, University of Colorado Health Sciences Center, 4200 E. 9th Avenue, Denver, CO 80262.

Received January 17, 2000; revised March 22, 2000; accepted March 24, 2000. at death, and was reversible, with binding levels returning to control values in subjects who had quit smoking for at least two months prior to death (Breese et al. 1997a). The up-regulation of receptor numbers does not appear to be under transcriptional regulation (Marks et al. 1992). Rather, the increases may be related to changes in receptor turnover based on receptor desensitization, subunit composition, secondary structural changes in the receptor, or to modification of the receptor by protein kinases (Peng et al. 1994; Baenziger and Chew 1997; Eilers et al. 1997; Flores et al. 1997; Hsu et al. 1997; Xiao et al. 1998; Fenster et al. 1999).

In spite of the high degree of nicotine self-administration in schizophrenics (Lohr and Flynn 1992; Ziedonis et al. 1994; de Leon et al. 1995), few studies have examined the effect of smoking or neuroleptic treatment on nicotinic receptor regulation in this disease (Dalack et al. 1998). It has been suggested that nicotine self-administration in schizophrenics may control a neuronal deficit. In this regard, it has been shown that abnormal electro- 
physiological and eye-tracking deficits can be normalized by cigarette smoking or nicotine administration (Adler et al. 1992, 1993; Olincy et al. 1998). Smoking may also alleviate neuroleptic-induced extrapyramidal side effects (Decina et al. 1990; Goff et al. 1992; Sandyk 1993). Nicotine use increases haloperidol metabolism (Jann et al. 1986; Miller et al. 1990), requiring patients that smoke to take higher doses than non-smoking schizophrenics, often further increasing their smoking behavior (McEvoy et al. 1995). Interestingly, clozapine, which does not induce the motor dysfunctions seen with typical neuroleptics, reduces smoking behavior (George et al. 1995) and normalizes the electrophysiological deficit in schizophrenics (Nagamoto et al. 1996).

Earlier studies from this laboratory have shown that schizophrenics have a reduced number of $\alpha$-bungarotoxin $(\alpha-\mathrm{BTX})$ binding sites in hippocampus, indicative of a reduction in the $\alpha-7$ neuronal nicotinic receptor (Freedman et al. 1995; Leonard et al. 1996, 1998a; Adler et al. 1998). Involvement of the $\alpha-7$ receptor in schizophrenia is further supported by genetic linkage to the region containing the $\alpha-7$ nicotinic receptor (Freedman et al. 1997; Leonard et al. 1998b; Kaufmann et al. 1998; Riley et al. 2000) and by animal studies showing that antagonists of the $\alpha-7$ nicotinic receptor induce sensory gating deficits similar to those seen in schizophrenia (Luntz-Leybman et al. 1992; Stevens et al. 1996, 1998). In the present study, the effect of smoking history on the regulation of the high affinity nicotinic receptor ligands, $\left[{ }^{3} \mathrm{H}\right]$-nicotine and $\left[{ }^{3} \mathrm{H}\right]$-epibatidine, and the low affinity receptor ligand, $\left[{ }^{3} \mathrm{H}\right]$-methyllycaconitine (MLA), was examined in several brain regions from normal control and schizophrenic subjects. An examination of the possible interaction of nicotine and neuroleptic treatment on nicotinic receptor regulation was performed in rats chronically treated with haloperidol, nicotine and the combination of these drugs.

\section{MATERIALS AND METHODS}

\section{Human Postmortem Brain Collection and Storage}

Human brains were collected at autopsy following family donation. Hospital and autopsy records were reviewed, and family members and physicians interviewed to detail the age, sex, race, cause of death, mental illnesses, as well as cigarette, alcohol, and drug use. Subject parameters are shown in Table 1. After the brain was weighed and examined for gross pathology, it was divided sagitally and one hemisphere was preserved in formalin for neuropathological analysis. The other hemisphere was sliced coronally into $1 \mathrm{~cm}$ slices, from which regions of interest were dissected in 1 gram blocks, frozen in dry ice snow, and packaged for storage at $-75^{\circ} \mathrm{C}$ (Leonard et al. 1993).
In these studies, subjects with schizophrenia were compared to non-psychotic control patients. Patients with schizotypal and bipolar disorders were excluded from analysis. Schizophrenic subjects with verifiable medication histories were on typical antipsychotic medications (haloperidol and phenothiazine derivatives) except for subject SL163, who had been on clozapine for more than three years before death (see Table 1). Differing sample numbers used for binding studies in the various brain regions were a result of tissue availability.

\section{Tissue Homogenate Preparation}

Dissected regions of human postmortem hippocampus, cortex (Brodmann area 8/9), caudate, and thalamus were weighed and homogenized in 10 volumes of ice cold Krebs-Ringer's HEPES buffer $(118 \mathrm{mM} \mathrm{NaCl}, 4.8$ $\mathrm{mM} \mathrm{KCl}, 2.5 \mathrm{mM} \mathrm{CaCl}_{2}, 1.2 \mathrm{mM} \mathrm{MgSO}_{4}, 20 \mathrm{mM}$ HEPES, pH 7.5) in a Potter-Elvehjem homogenizer using a motor driven Teflon pestle (Breese et al. 1997a).

Three centrifugation steps $(30,000 \mathrm{~g})$ following homogenization or resuspension of pellet in $0.1 \times$ KrebsRinger's HEPES were included to provide thorough dissociation and washing of membranes. Following the final wash, pellets were resuspended in $0.1 \times$ KrebsRinger's HEPES buffer $(1 \mathrm{ml} / 100 \mathrm{mg}$ original wet weight), aliquoted, and assayed for protein content (BCA assay; Pierce, Rockford, IL). Membranes were stored at $-75^{\circ} \mathrm{C}$ until analyzed for $\left[{ }^{3} \mathrm{H}\right]$-nicotine and /or $\left[{ }^{3} \mathrm{H}\right]$-epibatidine binding. Although nicotine is the traditional ligand used for high affinity nicotinic receptor binding studies (Marks and Collins 1982; Breese et al. 1997a), we also used $\left[{ }^{3} \mathrm{H}\right]$-epibatidine, which had a higher affinity and lower non-specific binding similar to that seen in rat (Houghtling et al. 1995).

\section{L- $\left[{ }^{3} \mathrm{H}\right]$-Nicotine Binding}

$\left[{ }^{3} \mathrm{H}\right]$-Nicotine ([N-methyl $\left.{ }^{3} \mathrm{H}\right]$-nicotine, specific activity $82 \mathrm{Ci} / \mathrm{mmol}$; Amersham Corp., Arlington Heights, IL) was repurified in order to reduce nonspecific binding of the labeled ligand (Romm et al. 1990). [ $\left.{ }^{3} \mathrm{H}\right]-N i c o t i n e$ binding was measured at $4^{\circ} \mathrm{C}$, as previously described in hippocampal, thalamic, and cortical tissue (Breese et al. 1997a). Briefly, incubations were conducted at a volume of $100 \mu \mathrm{l}$ in 96-well polystyrene culture dishes. Samples containing 100-200 $\mu \mathrm{g}$ of protein were incubated at $4^{\circ} \mathrm{C}$ for $2 \mathrm{hrs}$ in Krebs-Ringers HEPES containing $200 \mathrm{mM}$ Tris. The binding reaction was terminated by filtration in an Inotech cell harvester apparatus (top filter: GB100 (Micro Filtration Systems, Dublin, CA); bottom filter: Type A/E (Gelman Sciences, Ann Arbor, Ml)), and filters counted on a Beckman LS3000 Liquid Scintillation Spectrometer (counting efficiency, $\approx 52 \%$ ).

Non-specific binding was established by inclusion of $10 \mu \mathrm{M}$ unlabeled nicotine in the incubation. Single-point 
assays were performed at a $\left[{ }^{3} \mathrm{H}\right]$-nicotine concentration of $12 \mathrm{nM}$. Scatchard analysis was performed on selected cortical samples by using serial dilutions of $\left[{ }^{3} \mathrm{H}\right]$-nicotine at concentrations ranging from $25 \mathrm{nM}$ to $1.56 \mathrm{nM}$.

\section{$\left[{ }^{3} \mathrm{H}\right]$-Epibatidine Binding}

$\left[{ }^{3} \mathrm{H}\right]$-epibatidine (specific activity $52 \mathrm{Ci} / \mathrm{mmol}$; Amersham Corp.) binding was performed as described above in cortex and caudate with the following modifications. Incubations were conducted at an incubation volume of $200 \mu \mathrm{l}$ in 96-well polystyrene culture dishes. Samples containing approximately $100-200 \mu \mathrm{g}$ of protein were incubated at room temperature for $2 \mathrm{hrs}$ in $0.25 \times$ Krebs-Ringers HEPES buffer. Blanks were established by inclusion of $250 \mu \mathrm{M}$ unlabeled nicotine in the incubation. Single-point assays were performed at a $\left[{ }^{3} \mathrm{H}\right]$-epibatidine concentration of $200 \mathrm{pM}$.

Scatchard analysis was performed on selected samples using serial dilutions of $\left[{ }^{3} \mathrm{H}\right]$-epibatidine at concentrations ranging from $1000 \mathrm{pM}$ to $7.8 \mathrm{pM}$. Once characterized in cortical samples, $\left[{ }^{3} \mathrm{H}\right]$-epibatidine binding was utilized in the remaining brain regions due to its higher specificity.

\section{[ $\left.{ }^{3} \mathrm{H}\right]-$ MLA Binding}

Levels of $\alpha-7$ neuronal nicotinic receptor binding were examined in cortex and hippocampus using $\left[{ }^{3} \mathrm{H}\right]$-methyllycaconitine $\left(\left[{ }^{3} \mathrm{H}\right]-\mathrm{MLA}\right.$; specific activity $26 \mathrm{Ci} /$ mmole; Tocris Cookson Ltd., Bristol, UK) (Davies et al. 1999). Total binding was done in $10 \mathrm{nM}\left[{ }^{3} \mathrm{H}\right]-\mathrm{MLA}$ and non-specific binding was defined in the presence of 1 $\mathrm{mM}$ nicotine. Samples were incubated $2 \mathrm{hrs}$ at room temperature, then filtered as described.

\section{Chronic Nicotine and Haloperidol Treatment in Rats}

A rat model was used to examine nicotinic receptor regulation in a controlled experimental paradigm of chronic neuroleptic and nicotine exposure. Animals were housed two per cage on a 12:12 light:dark cycle (lights on at $0700 \mathrm{hrs)}$ and allowed access to food and water ad libitum. Four groups of rats ( $n=10$ per group) were injected twice daily (0800 and $1800 \mathrm{hrs}$ ) with saline, $1.25 \mathrm{mg} / \mathrm{kg}$ nicotine (total dose $2.5 \mathrm{mg} / \mathrm{kg} /$ day), $1 \mathrm{mg} / \mathrm{kg}$ haloperidol (total dose 2 $\mathrm{mg} / \mathrm{kg} /$ day), or the combination of nicotine and haloperidol for six weeks. Eight hours after the last treatment, animals were sacrificed by $\mathrm{CO}_{2}$ inhalation, the brains rapidly removed, and the cortical mantel dissected and stored at $-75^{\circ} \mathrm{C}$ until tissue homogenization and receptor binding assays were performed, as described above.

\section{Data Analysis}

Specific binding (fmoles/mg protein) was calculated for each ligand. In studies using human postmortem brain tissue, correlational analyses were performed with age, sex, PMI, storage time, drug and alcohol histories in order to examine the possible role of these variables on the results. All data were analyzed using ANOVA by comparing the nicotine or epibatidine binding levels in schizophrenic and control subjects in adult nonsmokers, smokers at the time of death, and smokers who had quit at least two months prior to death. Appropriate specific contrasts were performed in order to identify the sources of variance.

Rat data was analyzed by ANOVA, followed by appropriate specific contrast (Crunch statistical software, Oakland, CA) (Keppel 1991). A correlational analysis was also performed using smoking history, defined as the number of packs smoked per day at death, and specific ligand binding (Breese et al. 1997a). The saturation analysis was performed with Prizm (GraphPad Software Inc., San Diego, CA), using a non-linear regression analysis.

\section{RESULTS}

\section{Postmortem Brain Tissue Samples}

Neuropathological analysis revealed that tissue samples used in this study were free of neuropathological disorders. There was no statistical difference based on smoking histories between control and schizophrenic subjects $(1.57 \pm 0.02$ vs $1.9 \pm 0.4$ packs per day, $p=.17)$. There were no statistical differences or interactions for age of the subjects at death (average age, $55.4 \pm 1.7$ years), or tissue storage time (hippocampal and thalamic tissues average storage, $865 \pm 57$ days; cortex and caudate average storage, $1228 \pm 61$ days), when compared with either mental illness or smoking history.

While there was a significantly longer postmortem interval (PMI) in schizophrenic subjects $(18.32 \pm 1.49$ vs. $14.87 \pm 0.8, p=.03)$, there were no differences in PMI based on smoking history, nor was there an interaction based on mental illness and smoking history (both $p>$.25). Correlational and statistical analyses showed no differences between control subjects and subjects with alcohol use or depression histories; therefore, these subjects were further analyzed as non-psychotic control subjects.

Correlational analyses were performed using various subject and tissue collection parameters with nicotinic receptor binding in order to examine the potential influence of these variables on the data. The results are summarized for $\left[{ }^{3} \mathrm{H}\right]$-epibatidine binding in cortical tissues for postmortem interval (Figure 1, left), tissue storage time (Figure 1, center), and age (Figure 1, right). No significant correlations were found between PMI (all $p>$.17) or tissue storage time (all $p>.05$ ) for either $\left[{ }^{3} \mathrm{H}\right]$-nicotine or $\left[{ }^{3} \mathrm{H}\right]$-epibatidine binding in any region examined.

A modest negative correlation was found between nicotinic receptor binding and age in schizophrenic 
Table 1. Summary of Patient and Sample Data

\begin{tabular}{|c|c|c|c|c|c|c|c|c|c|c|c|c|c|c|c|}
\hline Subject & $\begin{array}{l}\text { Mental } \\
\text { Illness }\end{array}$ & Smoker & $\begin{array}{c}\text { Smoker } \\
\text { that } \\
\text { Quit }\end{array}$ & $\begin{array}{l}\text { Packs/ } \\
\text { Day }\end{array}$ & Alcohol & Sex & Race & Age & COD & PMI & Drugs & $\begin{array}{l}\text { Hippocampus } \\
\text { Binding }\end{array}$ & $\begin{array}{l}\text { Thalamus } \\
\text { Binding }\end{array}$ & $\begin{array}{c}\text { Cortex } \\
\text { Binding }\end{array}$ & $\begin{array}{l}\text { Caudate } \\
\text { Binding }\end{array}$ \\
\hline SB154 & Control & No & No & 0 & No & $\mathrm{M}$ & C & 65 & adis & 11 & $\mathrm{U}$ & 5.5 & & & \\
\hline SL051 & Control & No & No & 0 & No & $\mathrm{F}$ & C & 23 & suic & 12 & $\mathrm{U}$ & & & & 10.8 \\
\hline SL107 & Control & No & No & 0 & No & $\mathrm{M}$ & C & 59.9 & suic & 19 & $\mathrm{U}$ & 11.1 & 54.9 & 18.5 & 4.4 \\
\hline SL111 & Control & No & No & 0 & No & $\mathrm{M}$ & $\mathrm{C}$ & 76.7 & card & 14 & $\mathrm{~N}$ & 12.1 & 38.8 & 18.9 & 14.7 \\
\hline SL118 & Control & No & No & 0 & No & $\mathrm{M}$ & C & 40.4 & card & 21 & $\mathrm{U}$ & & & 15.9 & 17.0 \\
\hline SL119 & Control & No & No & 0 & No & $\mathrm{F}$ & C & 40.1 & card & 11.5 & $\mathrm{~N}$ & & & 16.6 & 9.4 \\
\hline SL125 & Control & No & No & 0 & No & $\mathrm{M}$ & C & 57.3 & card & 3 & $\mathrm{U}$ & & & 15.1 & 19.6 \\
\hline SL128 & Control & No & No & 0 & No & $\mathrm{M}$ & C & 80.3 & cdis & 22.5 & $\mathrm{~N}$ & 7.4 & 30.9 & 7.8 & 6.9 \\
\hline SL130 & Control & No & No & 0 & Yes & $\mathrm{M}$ & $\mathrm{H}$ & 19.7 & suic & 14 & $\mathrm{~N}$ & 14.1 & 53.4 & 26.3 & 14.1 \\
\hline SL136 & Control & No & No & 0 & No & $\mathrm{M}$ & C & 26.5 & card & 15.5 & U & 9.8 & 45.9 & 32.7 & 27.5 \\
\hline SL139 & Control & No & No & 0 & No & $\mathrm{M}$ & C & 37.6 & adis & 5 & $\mathrm{~N}$ & 18.9 & 30.5 & 29.2 & 8.2 \\
\hline SL144 & Control & No & No & 0 & No & $\mathrm{M}$ & C & 14.6 & suic & 14 & $\mathrm{~N}$ & 17.3 & 13.3 & 49.3 & 17.3 \\
\hline SL153 & Control & No & No & 0 & No & $\mathrm{F}$ & C & 41.7 & card & 8 & $\mathrm{~N}$ & 16.4 & 24.5 & 29.3 & \\
\hline SL164 & Control & No & No & 0 & No & $\mathrm{F}$ & B & 69.9 & card & 5.5 & $\mathrm{~N}$ & 6.8 & 22.4 & 20.9 & 5.9 \\
\hline SL165 & Control & No & No & 0 & No & $\mathrm{M}$ & C & 11.9 & suic & 15.5 & $\mathrm{~N}$ & 12.5 & 27.8 & 45.6 & 13.3 \\
\hline SL166 & Control & No & No & 0 & No & $\mathrm{F}$ & C & 39.3 & cdis & 24 & $\mathrm{~N}$ & 10.0 & 24.2 & 18.6 & 6.9 \\
\hline SL173 & Control & No & No & 0 & Yes & $\mathrm{M}$ & C & 59.4 & cdis & 21 & $\mathrm{~N}$ & & & 13.6 & 17.2 \\
\hline SL179 & Control & No & No & 0 & No & $\mathrm{M}$ & B & 41.7 & cdis & 19.5 & $\mathrm{~N}$ & & & 10.2 & 5.6 \\
\hline SL019 & Control & Yes & No & 0.5 & No & $\mathrm{M}$ & C & 71 & cdis & 9 & $\mathrm{~N}$ & 7.5 & & & \\
\hline SL116 & Control & Yes & No & 0.5 & No & $\mathrm{M}$ & C & 68 & cdis & 5.5 & $\mathrm{P}$ & 8.4 & 32.4 & 12.8 & 5.1 \\
\hline SB151 & Control & Yes & No & 1 & No & $\mathrm{M}$ & C & 59 & cdis & 24 & $\mathrm{U}$ & 16.3 & & & \\
\hline SL061 & Control & Yes & No & 1 & No & $\mathrm{M}$ & C & 24.3 & trma & 13 & $\mathrm{U}$ & & & 76.4 & 19.1 \\
\hline SL075 & Control & Yes & No & 1 & Yes & $\mathrm{F}$ & C & 62.1 & card & 18 & D & & & 52.9 & 30.6 \\
\hline SL085 & Control & Yes & No & 1 & No & $\mathrm{M}$ & C & 49.7 & card & 7 & $\mathrm{U}$ & 17.0 & 58.5 & 66.8 & 22.9 \\
\hline SL086 & Control & Yes & No & 1 & No & $\mathrm{F}$ & C & 56.8 & card & 18 & $\mathrm{~N}$ & & & 32.8 & 11.5 \\
\hline SL129 & Control & Yes & No & 1 & Yes & $\mathrm{F}$ & C & 32.3 & suic & 14.5 & $\mathrm{~N}$ & 18.6 & 47.7 & 38.3 & 43.2 \\
\hline SL147 & Control & Yes & No & 1 & No & $\mathrm{F}$ & C & 75.1 & cdis & 6.5 & $\mathrm{~N}$ & 8.4 & 42.7 & 26.5 & \\
\hline SL148 & Control & Yes & No & 1 & No & $\mathrm{F}$ & $\mathrm{C}$ & 49.6 & suic & 16.5 & $\mathrm{~N}$ & 25.9 & 59.3 & 63.6 & 39.6 \\
\hline SL155 & Control & Yes & No & 1 & No & $\mathrm{F}$ & C & 58.9 & resp & 9.5 & $\mathrm{~N}$ & & & 53.1 & 51.3 \\
\hline SL010 & Control & Yes & No & 1.5 & No & $\mathrm{M}$ & B & 41 & card & 7 & $\mathrm{~N}$ & 26.8 & & & \\
\hline SL062 & Control & Yes & No & 1.5 & Yes & $\mathrm{F}$ & C & 57.4 & adis & 15 & $\mathrm{U}$ & 26.9 & 36.2 & 60.0 & 19.4 \\
\hline SL084 & Control & Yes & No & 1.5 & Yes & $\mathrm{M}$ & C & 43.9 & card & 20 & $\mathrm{U}$ & 29.3 & 87.1 & 65.8 & 16.4 \\
\hline SL098 & Control & Yes & No & 1.5 & Yes & $\mathrm{M}$ & C & 26 & suic & 14 & $\mathrm{U}$ & 37.1 & 70.9 & 62.6 & 22.3 \\
\hline SL132 & Control & Yes & No & 1.5 & No & $\mathrm{M}$ & C & 50.5 & card & 6 & D & 27.9 & 71.9 & & 11.4 \\
\hline SL006 & Control & Yes & No & 2 & No & $\mathrm{M}$ & C & 61 & card & 20 & $\mathrm{~N}$ & 19.5 & & & \\
\hline SL007 & Control & Yes & No & 2 & Yes & $\mathrm{M}$ & C & 79 & adis & 16 & $\mathrm{U}$ & 46.0 & & & \\
\hline SL097 & Control & Yes & No & 2 & Yes & $\mathrm{M}$ & C & 52.9 & adis & 20 & $\mathrm{~N}$ & & & 11.6 & 6.9 \\
\hline SL123 & Control & Yes & No & 2 & No & $\mathrm{M}$ & C & 52.1 & adis & 5.5 & $\mathrm{~N}$ & 30.8 & 65.6 & & 23.7 \\
\hline SL152 & Control & Yes & No & 2 & No & $\mathrm{M}$ & C & 46.7 & card & 18 & $\mathrm{~N}$ & & & 74.3 & 39.5 \\
\hline SL174 & Control & Yes & No & 2 & No & $\mathrm{M}$ & C & 21.7 & trma & 9.5 & $\mathrm{~N}$ & & & 35.4 & 29.0 \\
\hline SL175 & Control & Yes & No & 2 & Yes & M & C & 56.3 & cdis & 12 & $\mathrm{P}$ & & & 31.0 & 32.2 \\
\hline SL181 & Control & Yes & No & 2 & No & $\mathrm{M}$ & C & 38 & suic & 23 & $\mathrm{~N}$ & & & 30.0 & 17.2 \\
\hline SL158 & Control & Yes & No & 2.2 & Yes & $\mathrm{F}$ & C & 67.3 & card & 5.5 & $\mathrm{~N}$ & 30.6 & 71.2 & 51.0 & 24.2 \\
\hline SL112 & Control & Yes & No & 2.25 & No & $\mathrm{M}$ & C & 72.7 & card & 11 & B & 17.8 & 46.2 & 39.9 & 12.2 \\
\hline SL142 & Control & Yes & No & 2.5 & Yes & $\mathrm{M}$ & C & 59.9 & suic & 17.5 & $\mathrm{~N}$ & 22.9 & 48.3 & 64.4 & \\
\hline SL087 & Control & Yes & No & 3.5 & Yes & $\mathrm{M}$ & C & 43.3 & card & 22 & B & 27.4 & 44.7 & 87.0 & 47.1 \\
\hline SL168 & Control & Yes & Yes & 0.25 & No & $\mathrm{M}$ & C & 75.8 & adis & 19.5 & D & 3.4 & 20.0 & 8.5 & \\
\hline SL021 & Control & Yes & Yes & 0.33 & No & $\mathrm{M}$ & $\mathrm{H}$ & 71 & cdis & 19 & $\mathrm{~N}$ & 4.2 & & & \\
\hline SL113 & Control & Yes & Yes & 0.5 & Yes & M & C & 83.3 & resp & 7 & $\mathrm{~N}$ & & & 15.2 & 9.5 \\
\hline SL016 & Control & Yes & Yes & 0.75 & Yes & $\mathrm{M}$ & B & 70 & cdis & 33 & $\mathrm{P}$ & 6.8 & & & \\
\hline SL133 & Control & Yes & Yes & 0.75 & No & $\mathrm{M}$ & C & 51.7 & card & 17.5 & $\mathrm{~N}$ & 13.6 & 53.8 & 23.6 & 13.4 \\
\hline SL140 & Control & Yes & Yes & 0.75 & No & M & C & 45 & card & 5 & $\mathrm{~N}$ & 11.0 & 55.9 & 33.6 & 15.0 \\
\hline SL082 & Control & Yes & Yes & 1 & No & $\mathrm{F}$ & C & 63 & cdis & 21 & $\mathrm{~N}$ & & & 17.4 & 22.1 \\
\hline SL167 & Control & Yes & Yes & 1 & No & $\mathrm{M}$ & C & 66 & adis & 22.5 & D & 6.3 & 24.9 & 21.4 & 11.1 \\
\hline SL172 & Control & Yes & Yes & 1 & No & $\mathrm{M}$ & C & 73.7 & cdis & 5.5 & $\mathrm{D}$ & & & 11.7 & 15.8 \\
\hline SL177 & Control & Yes & Yes & 1 & Yes & $\mathrm{M}$ & C & 72.1 & cdis & 25.5 & D & & & 8.0 & 4.9 \\
\hline SL008 & Control & Yes & Yes & 1.5 & No & M & C & 66 & card & 12 & $\mathrm{U}$ & 10.0 & & & \\
\hline SL160 & Control & Yes & Yes & 1.5 & Yes & $\mathrm{M}$ & C & 57.1 & card & 18 & $\mathrm{~N}$ & 6.8 & 29.6 & 13.8 & \\
\hline SL093 & Control & Yes & Yes & 2 & Yes & $\mathrm{M}$ & C & 60.1 & cdis & 24 & $\mathrm{~N}$ & & & 13.9 & 17.2 \\
\hline SL101 & Control & Yes & Yes & 2 & Yes & $\mathrm{M}$ & C & 70 & resp & 21 & $\mathrm{D}$ & & & 17.0 & 13.4 \\
\hline SL169 & Control & Yes & Yes & 2 & No & $\mathrm{M}$ & C & 73 & renl & 22 & D & & & 13.9 & 6.1 \\
\hline SL091 & Control & Yes & Yes & 3 & No & $\mathrm{M}$ & C & 58.2 & card & 18 & $\mathrm{~N}$ & & & 12.5 & \\
\hline SL053 & Depression & No & No & 0 & No & $\mathrm{F}$ & $\mathrm{H}$ & 42 & card & 20 & $\mathrm{D}$ & & & & 36.7 \\
\hline SL096 & Depression & No & No & 0 & No & $\mathrm{M}$ & C & 50.5 & card & 24 & B & 9.9 & 41.8 & 18.0 & 16.2 \\
\hline
\end{tabular}


Table 1. (continued)

\begin{tabular}{|c|c|c|c|c|c|c|c|c|c|c|c|c|c|c|c|}
\hline Subject & $\begin{array}{l}\text { Mental } \\
\text { Illness }\end{array}$ & Smoker & $\begin{array}{c}\text { Smoker } \\
\text { that } \\
\text { Quit }\end{array}$ & $\begin{array}{l}\text { Packs/ } \\
\text { Day }\end{array}$ & Alcohol & Sex & Race & Age & COD & PMI & Drugs & $\begin{array}{l}\text { Hippocampus } \\
\text { Binding }\end{array}$ & $\begin{array}{c}\text { Thalamus } \\
\text { Binding }\end{array}$ & $\begin{array}{l}\text { Cortex } \\
\text { Binding }\end{array}$ & $\begin{array}{l}\text { Caudate } \\
\text { Binding }\end{array}$ \\
\hline SL121 & Depression & No & No & 0 & No & $\mathrm{M}$ & C & 69.9 & adis & 11 & B & & & 13.4 & 8.6 \\
\hline SL122 & Depression & No & No & 0 & No & $\mathrm{M}$ & C & 73.5 & adis & 9.5 & B & & & 9.8 & 25.0 \\
\hline SL126 & Depression & No & No & 1 & No & $\mathrm{F}$ & C & 52.8 & card & 4.5 & $\mathrm{D}$ & 32.3 & 83.4 & 61.9 & 14.5 \\
\hline SL178 & Depression & Yes & No & 1 & Yes & $\mathrm{M}$ & C & 50.4 & adis & 13 & $\mathrm{~N}$ & & & 38.4 & 33.2 \\
\hline SL146 & Depression & Yes & No & 2.5 & No & $\mathrm{F}$ & C & 62.9 & card & 14 & $\mathrm{~N}$ & 16.2 & 40.9 & 38.6 & 8.4 \\
\hline SL055 & Depression & Yes & Yes & 1 & No & $\mathrm{F}$ & C & 44.2 & adis & 7 & $\mathrm{D}$ & 14.8 & 21.3 & 16.1 & 6.7 \\
\hline SL042 & Depression & Yes & Yes & 2 & & $\mathrm{M}$ & C & 61 & cdis & 23 & $\mathrm{U}$ & & & & 9.6 \\
\hline SL104 & Schizophrenia & No & No & 0 & No & $\mathrm{M}$ & C & 59.9 & resp & 24 & B & 10.4 & 61.2 & 15.8 & 7.6 \\
\hline SL110 & Schizophrenia & No & No & 0 & No & $\mathrm{M}$ & C & 63.5 & card & 18 & $\mathrm{U}$ & 29.3 & 77.2 & 17.2 & 11.0 \\
\hline SL114 & Schizophrenia & No & No & 0 & No & $\mathrm{F}$ & C & 45.5 & card & 31 & $\mathrm{P}$ & & & 20.2 & 17.3 \\
\hline SL127 & Schizophrenia & No & No & 0 & Yes & $\mathrm{M}$ & $\mathrm{C}$ & 38.6 & adis & 11 & $\mathrm{~N}$ & 14.2 & 65.8 & 15.8 & 23.1 \\
\hline SL154 & Schizophrenia & No & No & 0 & No & $\mathrm{F}$ & C & 47.1 & resp & 26.5 & B & 8.1 & 29.2 & 16.6 & 9.8 \\
\hline SL162 & Schizophrenia & No & No & 0 & No & $\mathrm{F}$ & C & 86.7 & resp & 19.5 & $\mathrm{U}$ & 11.6 & 32.4 & 24.7 & \\
\hline SL026 & Schizophrenia & Yes & No & 0.25 & No & $\mathrm{M}$ & $\mathrm{U}$ & 77 & unk & 4 & $\mathrm{P}$ & 5.3 & & 8.3 & 7.3 \\
\hline SL022 & Schizophrenia & Yes & No & 0.27 & No & $\mathrm{F}$ & B & 65 & adis & 22 & $\mathrm{D} / \mathrm{L}$ & 11.3 & & 27.5 & \\
\hline SL157 & Schizophrenia & Yes & No & 0.7 & No & $\mathrm{F}$ & C & 72.1 & cdis & 18 & $\mathrm{P}$ & 11.7 & 37.2 & 25.8 & 14.3 \\
\hline SL015 & Schizophrenia & Yes & No & 1 & No & $\mathrm{M}$ & C & 59 & adis & 20 & B & 6.2 & & 12.2 & 4.5 \\
\hline SL083 & Schizophrenia & Yes & No & 1 & No & $\mathrm{M}$ & C & 69.8 & card & 15 & $\mathrm{~N}$ & 16.5 & 56.4 & 24.7 & \\
\hline SL102 & Schizophrenia & Yes & No & 1 & No & $\mathrm{M}$ & C & 57.1 & cdis & 3 & B & 10.2 & 47.7 & 18.5 & 3.4 \\
\hline SL115 & Schizophrenia & Yes & No & 1 & No & $\mathrm{F}$ & C & 51.1 & card & 15 & $\mathrm{P}$ & 20.9 & 22.0 & 51.2 & 16.7 \\
\hline SL151 & Schizophrenia & Yes & No & 1 & No & M & A & 36 & suic & 28 & U & & & 54.7 & 13.6 \\
\hline SL120 & Schizophrenia & Yes & No & 1.5 & No & $\mathrm{M}$ & C & 67.3 & resp & 17 & $P$ & 16.6 & 63.7 & 22.5 & \\
\hline SL056 & Schizophrenia & Yes & No & 2 & No & $\mathrm{F}$ & C & 69.3 & card & 14 & $\mathrm{P}$ & & & 42.2 & 14.5 \\
\hline SL106 & Schizophrenia & Yes & No & 2 & No & $\mathrm{F}$ & C & 30 & resp & 4.5 & $\mathrm{P}$ & & & 8.8 & 8.9 \\
\hline SL135 & Schizophrenia & Yes & No & 2 & No & $\mathrm{F}$ & $\mathrm{H}$ & 57.7 & cdis & 12 & B & 26.5 & 60.2 & 62.7 & 13.7 \\
\hline SL149 & Schizophrenia & Yes & No & 2 & No & $\mathrm{F}$ & B & 36.5 & card & 14.5 & B & & & 27.2 & 27.8 \\
\hline SL018 & Schizophrenia & Yes & No & 2.5 & No & $\mathrm{M}$ & C & 40 & suic & 20 & B & 32.5 & & 86.8 & \\
\hline SL105 & Schizophrenia & Yes & No & 2.5 & No & $\mathrm{M}$ & C & 49.9 & card & 30 & $\mathrm{~N}$ & 12.7 & 50.6 & 38.8 & 11.0 \\
\hline SL145 & Schizophrenia & Yes & No & 2.5 & No & $\mathrm{M}$ & C & 60.8 & cdis & 23.5 & $\mathrm{U}$ & 23.0 & 24.4 & 64.6 & 16.4 \\
\hline SL094 & Schizophrenia & Yes & No & 3 & Yes & $\mathrm{F}$ & C & 35.1 & adis & 2 & $\mathrm{P}$ & & & 35.1 & 11.3 \\
\hline SL103 & Schizophrenia & Yes & No & 3 & No & $\mathrm{F}$ & C & 39.4 & card & 30 & B & & & 47.6 & 27.3 \\
\hline SL141 & Schizophrenia & Yes & No & 3 & No & $\mathrm{F}$ & $\mathrm{C}$ & 50.4 & card & 24 & $\mathrm{P}$ & 28.7 & 66.6 & 42.9 & 25.4 \\
\hline SL143 & Schizophrenia & Yes & No & 3 & Yes & $\mathrm{M}$ & C & 67.7 & adis & 23 & $\mathrm{P}$ & & & 19.3 & 2.5 \\
\hline SL159 & Schizophrenia & Yes & No & 3 & No & $\mathrm{M}$ & C & 75.7 & cdis & 26 & $\mathrm{P}$ & & & 24.5 & 30.3 \\
\hline SL163 & Schizophrenia & Yes & No & 3.5 & No & $\mathrm{M}$ & $\mathrm{C}$ & 38.9 & adis & 27 & $\mathrm{P}^{*}$ & 20.5 & 33.6 & 41.5 & 15.4 \\
\hline SL156 & Schizophrenia & Yes & Yes & 0.5 & No & $\mathrm{M}$ & $\mathrm{C}$ & 95.5 & cdis & 6.5 & $\mathrm{P}$ & 7.3 & 39.4 & 9.0 & 10.5 \\
\hline SL003 & Schizophrenia & Yes & Yes & 2 & No & $\mathrm{M}$ & C & 73 & card & 22 & B & 17.0 & & 13.9 & 14.1 \\
\hline SL138 & Schizophrenia & Yes & Yes & 2.5 & No & $\mathrm{M}$ & C & 85.5 & cdis & 17 & B & 8.6 & 43.9 & 10.6 & 3.3 \\
\hline
\end{tabular}

Table shows ${ }^{3} \mathrm{H}$-nicotine binding in hippocampus and thalamus and ${ }^{3} \mathrm{H}$-epibatidine binding in cortex and caudate. Data listing for a subject indicate samples also used for ${ }^{3} \mathrm{H}$-methyllycaconitine binding in hippocampus and ${ }^{3} \mathrm{H}$-nicotine and ${ }^{3} \mathrm{H}$-methyllycaconitine binding in cortex.

Abbreviations: $\mathrm{COD}=$ cause of death (see below for key); $\mathrm{PMI}=$ postmortem interval; Drugs: Known medications at time of death.

Race: $\mathrm{C}=$ Caucasian, $\mathrm{B}=$ Black, $\mathrm{H}=$ Hispanic, $\mathrm{A}=$ Asian, $\mathrm{U}=$ Undetermined. COD (cause of death): cdis = chronic disease, adis $=$ acute disease, card $=$ cardiac arrest, suic $=$ suicide, trma $=$ trauma, resp $=$ respiratory disease, renl $=$ renal failure. Drugs: $\mathrm{P}=$ history of antipsychotic drug use $\left({ }^{*}\right.$ clozapine $), \mathrm{D}=$ history of antidepressant drug use, $\mathrm{B}=$ history of antipsychotic and antidepressant drug use, $\mathrm{N}=$ no drugs in these classes at time of death, $\mathrm{L}=\mathrm{lithium}, \mathrm{U}=$ unverified drug status at time of death.

subjects in hippocampus and cortex, and in control subjects in cortex (Figure 1, right) (cortex: controls $\mathrm{r}=$ $-0.405, p<.002$; schizophrenics $\mathrm{r}=-0.39, p<.04$; hippocampus (data not shown): schizophrenics $r=-0.46$, $p<.04)$. Age was not significantly correlated with either $\left[{ }^{3} \mathrm{H}\right]$-nicotine binding in thalamus or $\left[{ }^{3} \mathrm{H}\right]$-epibatidine binding in caudate (both $p>.10$ ).

\section{Binding of $\left[{ }^{3} \mathrm{H}\right]$-Epibatidine in Cortical Membranes of Control Subjects with Variable Smoking Histories}

Cortical membranes were used to characterize $\left[{ }^{3} \mathrm{H}\right]-$ epibatidine binding responses to tobacco use in human postmortem brain tissue. As shown in the binding curves of Figure 2, $\left[{ }^{3} \mathrm{H}\right]$-epibatidine binding increased with increasing smoking history (packs smoked per day at the time of death). The binding curves demonstrate that $\left[{ }^{3} \mathrm{H}\right]$-epibatidine binding was saturable in human postmortem brain tissues.

The correlation in cortex between $\left[{ }^{3} \mathrm{H}\right]$-nicotine and $\left[{ }^{3} \mathrm{H}\right]$-epibatidine binding in the same subject sample was $0.921(p<.0001)$ and yielded similar statistical results as the $\left[{ }^{3} \mathrm{H}\right]$-nicotine binding data. This suggests that $\left[{ }^{3} \mathrm{H}\right]$-epibatidine was measuring the same binding site as $\left[{ }^{3} \mathrm{H}\right]$-nicotine; however, $\left[{ }^{3} \mathrm{H}\right]$-epibatidine binding levels tended to be $\approx 20 \%$ higher than $\left[{ }^{3} \mathrm{H}\right]$-nicotine binding levels within the same sample. This may be related to the binding kinetics of these ligands for the nicotinic receptor site, although the potential for the measurement of additional nicotinic receptor sites cannot be ruled out (Houghtling et al. 1995; Marks et al. 1998). 

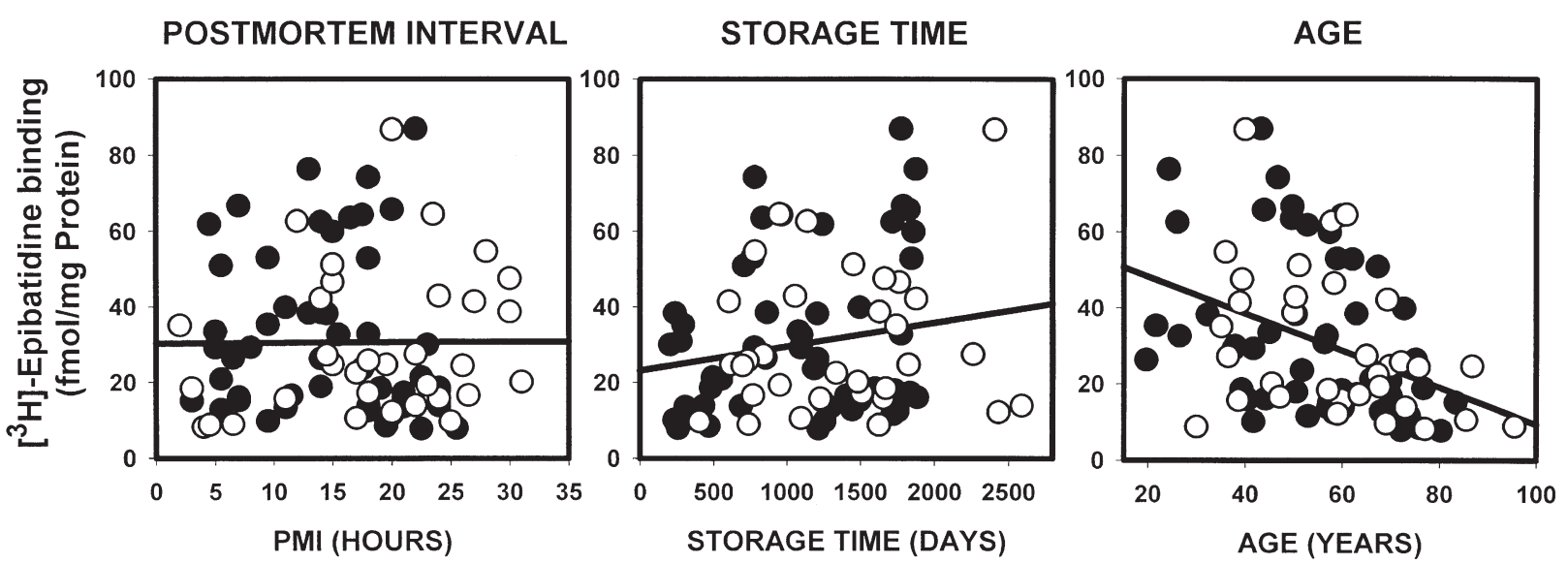

Figure 1. Correlational analysis of post mortem interval (PMI, left), and freezer storage time (middle) and subject age (right), with $\left[{ }^{3} \mathrm{H}\right]$-epibatidine binding levels in cortex. PMI and storage time had no statistically significant effect on the levels $\left[{ }^{3} \mathrm{H}\right]$-epibatidine binding in the sample population. A modest negative correlation was found between nicotinic receptor binding and age in cortex (controls, closed circles: $\mathrm{r}=-0.405, p<.002$; schizophrenics, open circles: $\mathrm{r}=-0.39, p<.04$ ).

$\left[{ }^{3} \mathrm{H}\right]$-epibatidine and $\left[{ }^{3} \mathrm{H}\right]$-nicotine results in cortex are directly compared below and were used to further characterize nicotinic receptor levels in human postmortem cortex and caudate.

\section{$\left[{ }^{3} \mathrm{H}\right]-N i c o t i n e$ Binding in Hippocampus, Thalamus, and Cortex of Normal Control and Schizophrenic Subjects}

Results of single point $\left[{ }^{3} \mathrm{H}\right]$-nicotine binding data in hippocampus, thalamus, and cortex are summarized in Figure 3. As originally described in a smaller group of subjects (Breese et al. 1997a), control smokers had significantly increased mean $\left[{ }^{3} \mathrm{H}\right]$-nicotine binding compared to either nonsmokers or smokers who had quit in both hippocampus (Figure 3, upper left; $n=42$, both $p<.0001$ ) and thalamus (Figure 3, upper right; $n=34$, $p<.01)$. This increase in nicotinic receptor levels in the human postmortem brains of smokers was replicated in a larger subject population using cortical tissues $(n=$

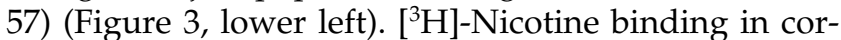
tex of normal control smokers was significantly increased compared to nonsmokers and demonstrated reversibility in smokers who had quit (both $p<.0001$ ).

In schizophrenic subjects, there was no such relationship between smoking history and $\left[{ }^{3} \mathrm{H}\right]$-nicotine receptor binding in any of these tissues. In hippocampus (Figure 3, upper left; $n=22 ; p=.45$ ) and thalamus (Figure 3 , upper right; $n=17 ; p=.67$ ), there were no differences between schizophrenic nonsmokers, smokers, or smokers who had quit. The cortex $(n=31)$ was the only region which showed a marginally significant difference in $\left[{ }^{3} \mathrm{H}\right]$-nicotine binding between schizophrenic nonsmokers and smokers (Figure 3, lower left; $p<.05$ ).

When control smokers were compared directly to schizophrenic smokers, schizophrenic smokers had signif-
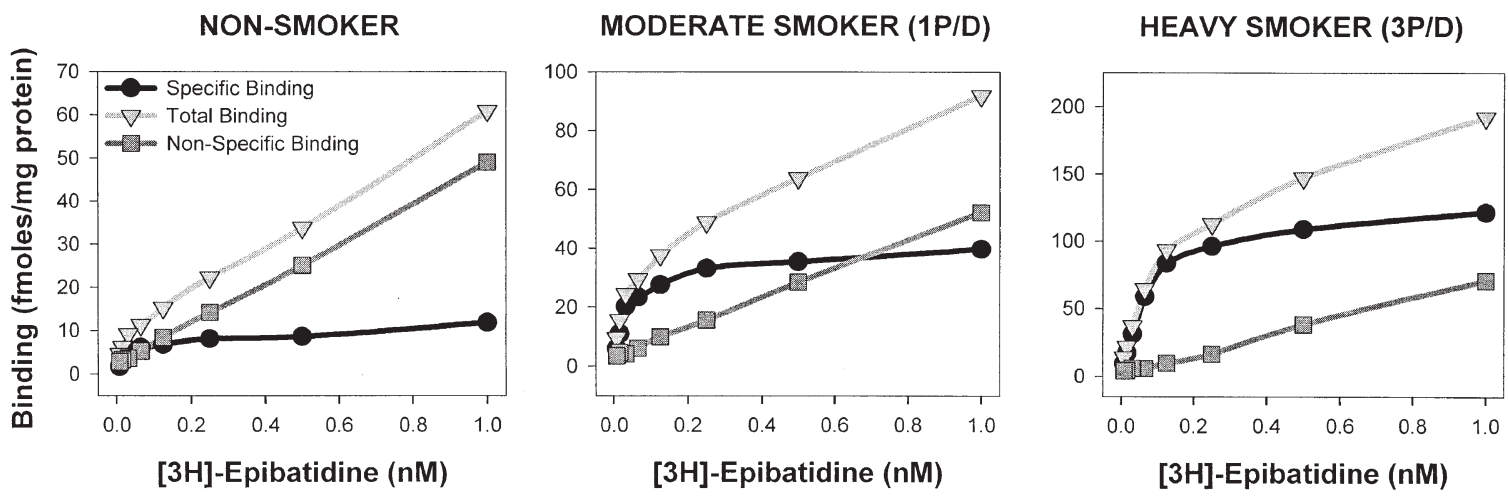

Figure 2. Binding of $\left[{ }^{3} \mathrm{H}\right]$-epibatidine in cortical membranes of control subjects with increasing smoking histories. Data shows increasing $\left[{ }^{3} \mathrm{H}\right]$-epibatidine binding with increasing number of cigarettes smoked/day at the time of death. Binding curves demonstrate that $\left[{ }^{3} \mathrm{H}\right]$-epibatidine binding is saturable in human brain samples [Nonsmoker: SL128; Moderate smoker: SL083; Heavy smoker: SL087]. 


\section{HIPPOCAMPUS}

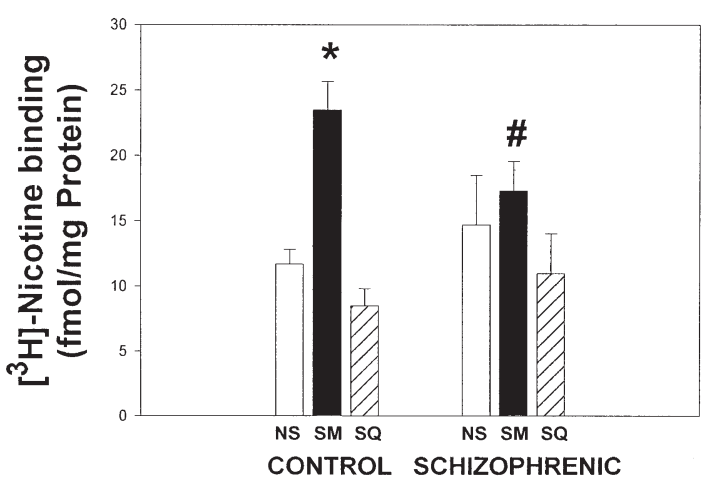

CORTEX

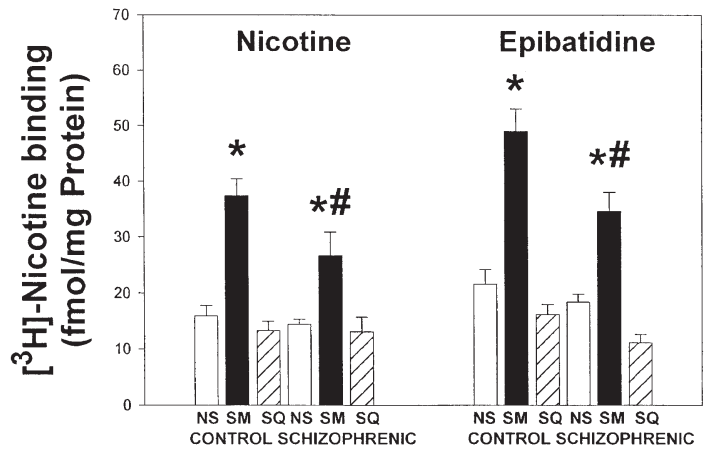

THALAMUS

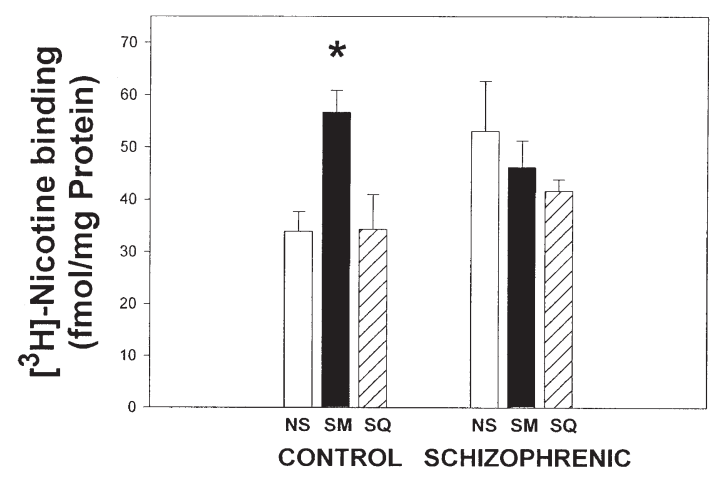

CAUDATE
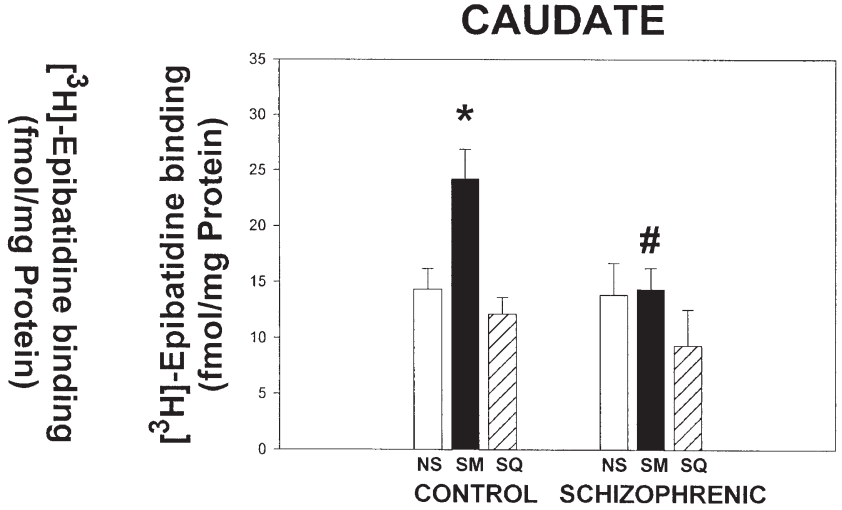

Figure 3. Single point-binding data for the hippocampus, thalamus, cortex, and caudate nucleus. In control smokers, a significant increase was observed in mean $\left[{ }^{3} \mathrm{H}\right]$-nicotine binding in smokers (S, filled bar) compared to either nonsmokers (NS, open bar) and smokers who had quit (SQ, cross-hatched bar) in hippocampus (upper left), thalamus (upper right), and cortex (lower left). A significant increase was also found in mean $\left[{ }^{3} \mathrm{H}\right]$-epibatidine binding in both the cortex and caudate nucleus of smokers compared to either nonsmokers, or smokers who had quit. In schizophrenic subjects, no such relationship between smoking history and nicotinic receptor levels was observed. In hippocampus, cortex, and caudate there was a significant reduction in nicotinic receptor numbers in schizophrenic smokers compared to control smokers (all $p<.05)$. The only brain region which showed a difference in nicotinic receptor levels between schizophrenic nonsmokers and smokers was in cortex (both $p<.05$; ${ }^{*}$ comparison between nonsmokers and smokers within the control or schizophrenic groups (all $p<.05)$; \# comparison between control smokers and schizophrenic smokers (all $p<.05)$ ).

icantly lower nicotinic receptor levels than control smokers in both hippocampus (Figure 3, upper left; $p<.05$ ) and cortex (Figure 3, lower left; $p<.03$ ), although there was no significant change in thalamus (Figure 3, upper right).

Since a significant correlation was observed between age and nicotinic receptor levels in both control and schizophrenic subjects in cortex, ANOVA was performed using age as a covariant in order to examine the potential effect of age on the reported results. In both subject groups co-varied for age, the overall results were similar to those described above, indicating that age was not a confounding variable in these analyses.

\section{$\left[{ }^{3} \mathrm{H}\right]$-Epibatidine Binding in the Cortex and Caudate of Normal Control and Schizophrenic Subjects}

$\left[{ }^{3} \mathrm{H}\right]$-epibatidine binding was performed in cortex and compared directly to cortical $\left[{ }^{3} \mathrm{H}\right]$-nicotine binding as shown in Figure 3 (lower left). In cortex, $\left[{ }^{3} \mathrm{H}\right]$-epibatidine binding was highly correlated with $\left[{ }^{3} \mathrm{H}\right]$-nicotine binding, as previously mentioned $(\mathrm{r}=0.925, p<.0001)$. $\left[{ }^{3} \mathrm{H}\right]$-epibatidine binding in cortex was increased in smokers compared to either nonsmokers or smokers who had quit (both $p<.0001$ ).

In the caudate nucleus of control subjects (Figure 3, lower right; $n=56$ ), $\left[{ }^{3} \mathrm{H}\right]$-epibatidine binding was also significantly increased in smokers compared to either nonsmokers or smokers who had quit (both $p<.001$ ). As with nicotine binding, there was also a difference in $\left[{ }^{3} \mathrm{H}\right]$-epibatidine binding in cortex between schizophrenic smokers and nonsmokers (Figure 3, lower left; $p<.03)$. In caudate $(n=26)$, there was no significant difference between schizophrenic nonsmokers, smokers, or smokers who quit (Figure 3, lower right; all $p>$ .50). In both tissues, schizophrenic smokers demonstrated significantly reduced levels of $\left[{ }^{3} \mathrm{H}\right]$-epibatidine 
binding when compared to control smokers (cortex: $p<$ .03 ; caudate: $p<.015)$.

\section{Comparison of $\left[{ }^{3} \mathrm{H}\right]$-Nicotine and $\left[{ }^{3} \mathrm{H}\right]$-Epibatidine Binding with Smoking History}

Since previous studies showed a dose-dependency in nicotinic receptor up-regulation (Breese et al. 1997a), differences in $\left[{ }^{3} \mathrm{H}\right]$-nicotine and $\left[{ }^{3} \mathrm{H}\right]$-epibatidine binding were further examined with regard to smoking history, as defined by the number of packs smoked per day at the time of death (Breese et al. 1997a). As shown in Figure 4, nicotinic receptor binding increased in normal control subjects with increasing numbers of packs of cigarettes smoked per day, with positive correlations in hippocampus $(\mathrm{r}=0.64, p<.0001)$, thalamus $(\mathrm{r}=$ $0.46, p<.02)$, cortex $(\mathrm{r}=0.64 p<.0001)$, and caudate $(\mathrm{r}=$ $0.38, p<.02)$.

In schizophrenic subjects, while there was a positive correlation of nicotinic receptor numbers in hippocampus $(\mathrm{r}=0.56, p<.02)$ and cortex $(\mathrm{r}=0.44, p<.02)$ with increasing smoking history, the slope of the regression lines were decreased $40 \%$ in hippocampus and $50 \%$ in cortex relative to normal smokers (hippocampus: normal slope $=6.58 \pm 1.4$; schizophrenic slope $=3.99 \pm 1.5$; cortex (nicotine binding): normal slope $=11.02 \pm 2.1 ;$ schizophrenic slope $=5.48 \pm$ 2.23).
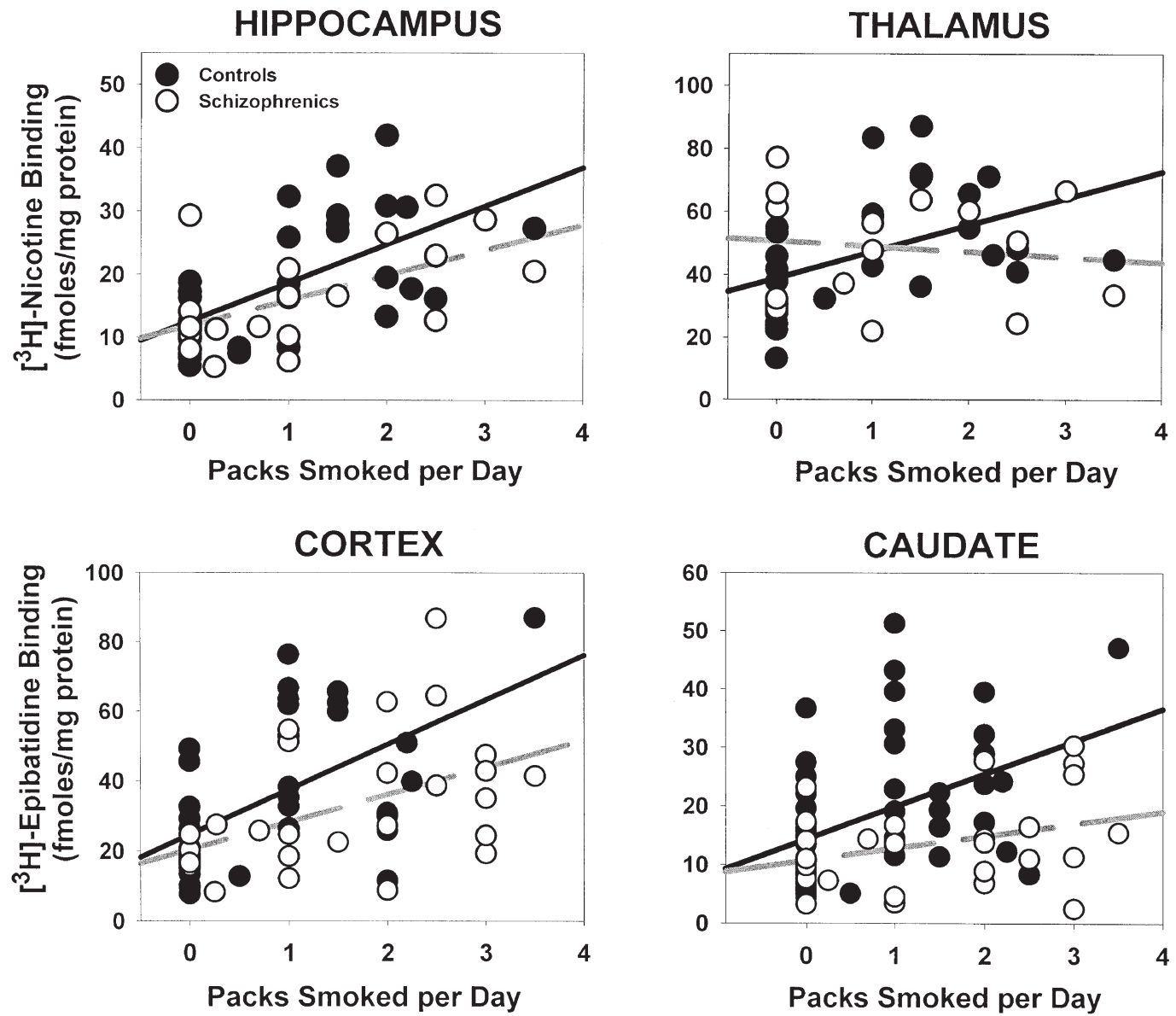

Figure 4. Correlational analysis of $\left[{ }^{3} \mathrm{H}\right]$-nicotine and $\left[{ }^{3} \mathrm{H}\right]$-epibatidine binding with the number of packs smoked per day at the time of death. In control subjects (filled circles and solid line) receptor number showed an increase with smoking history in all tissues examined (hippocampus: $\mathrm{r}=0.64, p<.0001$; thalamus: $\mathrm{r}=0.46, p<.02$; cortex: $\mathrm{r}=0.64, p<.0001$; caudate nucleus: $\mathrm{r}=0.38, p<.02$ ), indicating a dose-dependent increase in nicotinic binding levels in the brain of human smokers. While schizophrenic subjects (open circles) did demonstrate a dose dependent increase in hippocampal [ $\left.{ }^{3} \mathrm{H}\right]-$ nicotine binding $(\mathrm{r}=0.56, p<.02)$ and cortical $\left[{ }^{3} \mathrm{H}\right]$-nicotine $(\mathrm{r}=0.44, p<.02)$ and $\left[{ }^{3} \mathrm{H}\right]$-epibatidine binding $(\mathrm{r}=0.49, p<.01)$, the slope of the regression line (dashed line) was decreased to $39.4 \%$ of the rate of normal smokers in hippocampus. The slope of the regression line in the cortex was reduced $50.3 \%$ and $45.6 \%$ for nicotine and epibatidine binding (respectively) in schizophrenics compared to normal smokers. In contrast to the dose dependent increase observed in thalamus and caudate of normal smokers, no correlation was found in schizophrenics between nicotine dose and $\left[{ }^{3} \mathrm{H}\right]$-nicotine binding for either tissue (thalamus: $\mathrm{r}=-0.12, p=.68$; caudate nucleus: $\mathrm{r}=0.31, p=.15$ ). The slope of the regression line in the caudate nucleus was reduced $60.2 \%$ in schizophrenics compared to control subjects. 
Results from $\left[{ }^{3} \mathrm{H}\right]$-epibatidine binding in the cortex were nearly identical to those found for $\left[{ }^{3} \mathrm{H}\right]$-nicotine binding (normal: $\mathrm{r}=0.63, p<.0001$, slope $=14.4 \pm$ 2.8; schizophrenic: $\mathrm{r}=0.49, p<.01$, slope $=7.84 \pm$ $2.8 ; 44 \%$ reduction in slope of regression line). In thalamus and caudate, nicotinic receptors in schizophrenic subjects were not significantly correlated with smoking history (thalamus: $\mathrm{r}=-0.12, p=.68$; caudate: $\mathrm{r}=0.31, p=.15$ ). In caudate, the reduction in the slope of the regression line was 60\% (normal slope $=4.99 \pm 1.9 ;$ schizophrenic slope $=1.98 \pm$ 1.34). In thalamus, there appeared to be a general dysregulation of nicotinic receptor upregulation in schizophrenics (normal slope $=8.5 \pm 3.2$; schizophrenic slope $=-1.72 \pm 4.0$ ).

Also as shown in Figure 4, the intercepts for hippocampus, caudate, and cortex were nearly identical for both control and schizophrenic subjects. This, in addition to the fact that there were no differences between control and schizophrenic nonsmokers (all $p>.1$ ), indicated that normal control and schizophrenic subjects have approximately the same basal levels of receptors in these brain regions. This was not the case in the thalamus, in which schizophrenic nonsmokers at death had marginally higher basal levels than control subjects $(p<.05)$.

\section{Scatchard Analysis}

Scatchard analyses were performed in cortical tissues using both $\left[{ }^{3} \mathrm{H}\right]$-nicotine and $\left[{ }^{3} \mathrm{H}\right]$-epibatidine from selected control $(n=9)$ and schizophrenic $(n=15)$ subjects with varying smoking histories. Regression analyses of the Scatchard plot of the data fit a straight line, consistent with that expected for a single binding site (Figure 5).There were the expected increases in $\mathrm{B}_{\max }$ for both $\left[{ }^{3} \mathrm{H}\right]$-nicotine and $\left[{ }^{3} \mathrm{H}\right]$-epibatidine binding with increasing degree of smoking in control subjects, and the reduced level of receptor binding $\left(B_{\max }\right)$ for the same level of cigarette smoking in schizophrenic subjects (Figure 5).

There were no statistically significant differences or interactions in mean binding affinity when examined by smoking history $(p<.20)$ or mental illness $(p<.20)$ for either $\left[{ }^{3} \mathrm{H}\right]$-nicotine or $\left[{ }^{3} \mathrm{H}\right]$-epibatidine binding, and the calculated mean binding affinity for $\left[{ }^{3} \mathrm{H}\right]$-nicotine was $\mathrm{k}_{\mathrm{d}}=2.06 \pm 0.25 \mathrm{nM}$ and $\left[{ }^{3} \mathrm{H}\right]$-epibatidine was $\mathrm{k}_{\mathrm{d}}=$ $55.02 \pm 3.6 \mathrm{pM}$. Although binding affinity was not spe-
NICOTINE BINDING

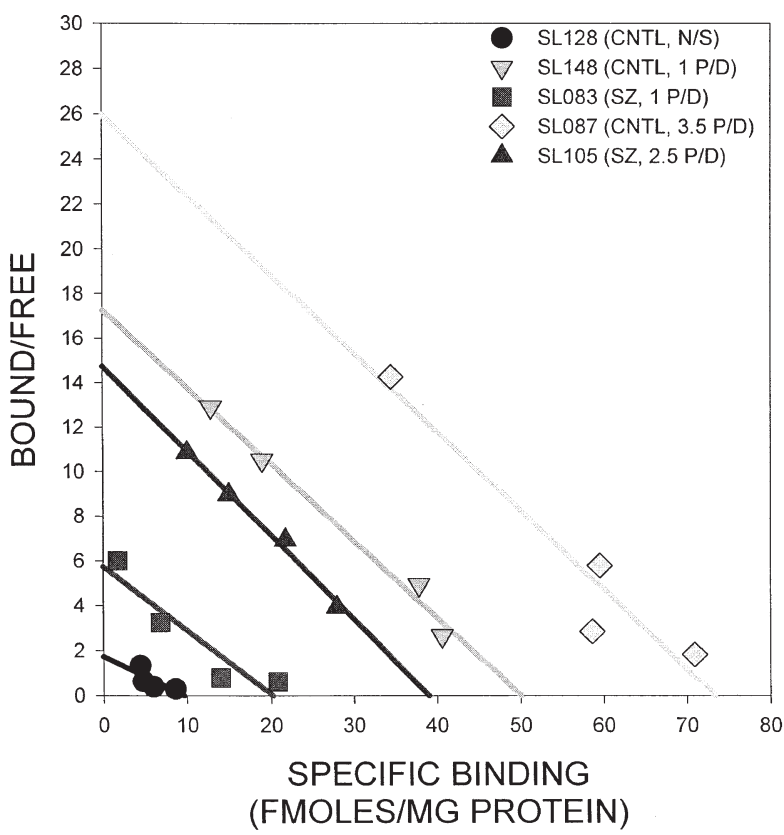

EPIBATIDINE BINDING

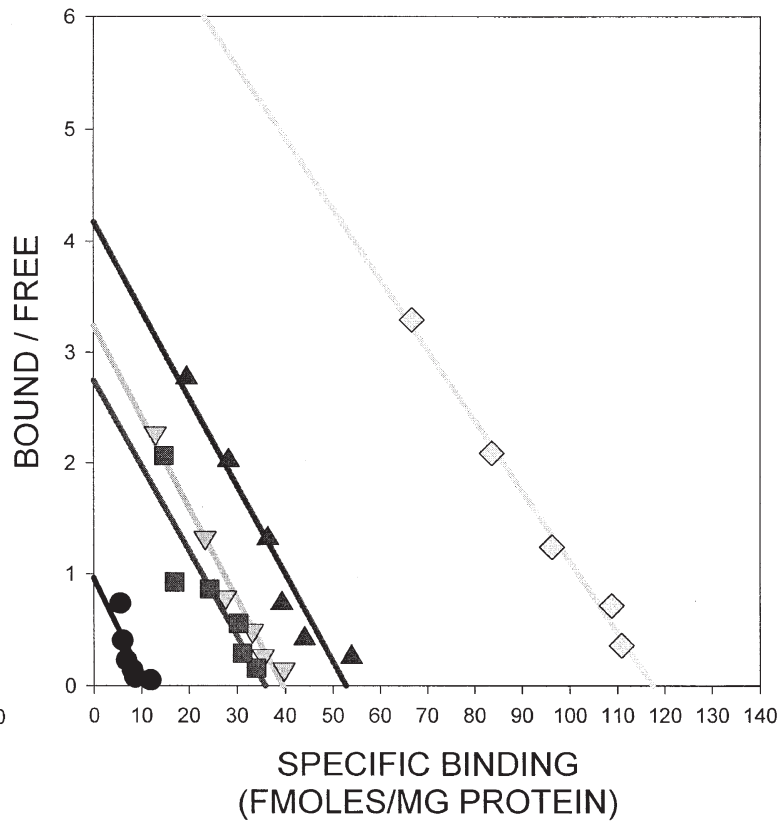

Figure 5. Scatchard analysis for $\left[{ }^{3} \mathrm{H}\right]$-nicotine and $\left[{ }^{3} \mathrm{H}\right]$-epibatidine binding in human cortex. Isolated membranes from human cortex were incubated with varying concentrations of $\left[{ }^{3} \mathrm{H}\right]$-nicotine and $\left[{ }^{3} \mathrm{H}\right]$-epibatidine. Scatchard plots are shown for control and schizophrenic subjects with different smoking histories (see legend). Schizophrenic subjects demonstrated an increase in receptor numbers with increased smoking history; however the increases were not as great as those observed in the control population. Parallelism of the lines indicates that smoking history did not correlate with any change in the affinity of nicotine or epibatidine for its receptor. Further analysis showed no differences or interactions in binding affinities when examined by smoking history $(p>.20)$ or mental illness $(p>.20)$ for either $\left[{ }^{3} \mathrm{H}\right]$-nicotine or $\left[{ }^{3} \mathrm{H}\right]$-epibatidine binding $(n=24)$. 
cifically examined in the other tissues used in this study, previous results in mice (Marks et al. 1985) and human postmortem brain (Benwell et al. 1988; Breese et al. 1997a) have shown that the receptor affinity for $\left[{ }^{3} \mathrm{H}\right]-$ nicotine binding was similar throughout the brain, and was not changed as a result of nicotine treatment or smoking history.

\section{$\left[{ }^{3} \mathrm{H}\right]-$ Methyllycaconitine Binding in Hippocampus and Cortex of Normal Control and Schizophrenic Subjects}

Low affinity nicotinic receptor binding for the $\alpha-7$ receptor was measured in cortex and hippocampus using $\left[{ }^{3} \mathrm{H}\right]$-methyllycaconitine (MLA). Receptor numbers measured with MLA were extremely low in both brain regions as previously described for $\left[{ }^{125} \mathrm{I}\right]-\alpha$-bungarotoxin binding (average $9.33 \pm 0.68$ ) (Freedman et al. 1995; Breese et al. 1997b). While schizophrenic subjects had reduced average levels of $\left[{ }^{3} \mathrm{H}\right]-M L A$ binding in both regions, no statistically significant differences were found in any of the brain regions examined (hippocampus: $9.882 \pm 0.73$ vs. $8.22 \pm 1.43, p=.25$; cortex: $10.31 \pm 0.77$ vs. $8.63 \pm 0.79, p=.16)$. There was no effect in either group for smoking history (all $p>.20$ ).

\section{Effect of Chronic Neuroleptic and Nicotine Treatment on $\left[{ }^{3} \mathrm{H}\right]$-Epibatidine Receptor Binding in Rats}

The effect of chronic nicotine, haloperidol, and co-treatment on $\left[{ }^{3} \mathrm{H}\right]$-epibatidine receptor binding in rat cortex is summarized in Figure 6. Rats treated with chronic haloperidol showed no change in $\left[{ }^{3} \mathrm{H}\right]$-epibatidine binding levels compared to saline treated control animals. Chronic nicotine treatment led to a significant increase in $\left[{ }^{3} \mathrm{H}\right]$-epibatidine binding in rat cortex compared to either saline or haloperidol treated animals $(p<.0001)$. Animals treated with the combination of nicotine and haloperidol showed a statistically significant increase in $\left[{ }^{3} \mathrm{H}\right]$-epibatidine binding levels in cortex compared to either saline or haloperidol treated animals $(p<.0001)$. This increase was not different from that observed with nicotine treatment alone.

\section{DISCUSSION}

Previous results have shown that the high affinity neuronal nicotinic receptors were up regulated in hippocampus and thalamus of subjects that smoke, compared to nonsmokers (Breese et al. 1997a). This increase was both dose-dependent and reversible. The present results extend these findings to cortex, a region sensitive to the regulatory effects of chronic nicotine treatment in rodents (Marks et al. 1985), and caudate, a region with a

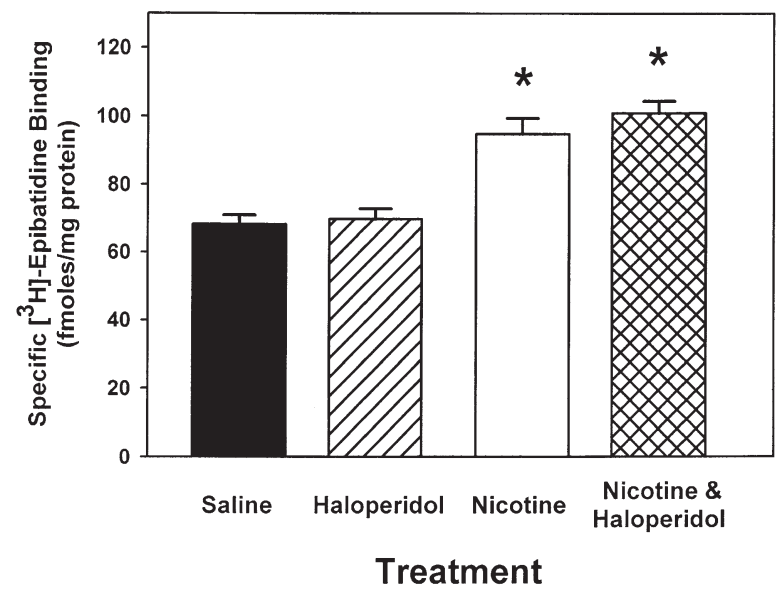

Figure 6. Effect of chronic nicotine, haloperidol, and haloperidol and nicotine treatment on $\left[{ }^{3} \mathrm{H}\right]$-epibatidine binding in the rat cortex. Rats treated with chronic haloperidol (single cross-hatched bar) showed no change in $\left[{ }^{3} \mathrm{H}\right]$-epibatidine binding from saline control animals (solid bar). Chronic nicotine treatment significantly increased $\left[{ }^{3} \mathrm{H}\right]$-epibatidine binding (open bar, ${ }^{*} p<.0001$ ), an increase not affected by the co-administration of haloperidol (double cross-hatched bar, ${ }^{*} p<.0001$ vs. saline control).

high density of dopaminergic terminals. In subjects with no history of mental illness, a significant increase in nicotinic receptor binding in these brain regions was seen in smokers compared to either nonsmokers or smokers who had quit. The increases were also correlated with packs smoked per day, further demonstrating the dose-dependency of nicotinic receptor up-regulation in human brain. Nicotinic receptor binding in smokers who had quit was comparable to nonsmokers, showing the reversibility of nicotinic receptor up-regulation in these brain regions when tobacco use is discontinued (Breese et al. 1997a). Scatchard analysis further indicated that these changes were related to changes in receptor number since the binding affinity of the neuronal nicotinic receptors was not changed as a result of smoking history (Breese et al. 1997a).

In the present study, schizophrenic subjects did not demonstrate the same regulatory responses for the high affinity neuronal nicotinic receptors as seen in control smokers. Schizophrenics who smoked demonstrated an overall reduction in the normal up-regulation of high affinity neuronal nicotinic receptors in most brain regions examined. While basal binding levels were not different between control and schizophrenic subjects, the reduction in high affinity nicotinic receptor binding in schizophrenic smokers was observed in group means, as well as in the regression analysis. The slope of the regression line, based on nicotinic receptor binding and number of cigarettes smoked per day, was reduced up to $60 \%$ in schizophrenic subjects, indicating a failure to up-regulate the high affinity neuronal nico- 
tinic receptors at any given smoking level compared to controls. Scatchard analyses suggested that the changes seen in nicotinic receptor levels were due to changes in total receptor numbers $\left(\mathrm{B}_{\max }\right)$ and not to a change in receptor affinity $\left(\mathrm{K}_{\mathrm{d}}\right)$.

Since schizophrenics are primarily treated with drugs that block dopaminergic neurotransmission, it was of interest to examine brain regions with dopaminergic innervation, namely striatum and cortex. In this study, caudate was examined as a representative region of striatum for measuring changes in high affinity neuronal nicotinic receptor binding in control smokers and schizophrenic subjects. Caudate, as well as cortex, showed a reduced level of nicotinic receptor upregulation in schizophrenics, compared to control subjects, suggesting that cholinergic modulation of dopaminergic neurotransmission might be affected in schizophrenia (Grenhoff and Svensson 1988; Rapier et al. 1990; Rowell 1995; Blaha et al. 1996; Pidoplichko et al. 1997). It is also interesting to note that schizophrenics demonstrated age related changes in neuronal nicotinic receptor numbers in both cortex and hippocampus, whereas control subjects demonstrated changes only in the cortex.

Previous results suggest that schizophrenics may have a loss of cognitive function (Hyde et al. 1994; Goldstein et al. 1998; Heinrichs and Zakzanis 1998), and an accelerated loss of nicotinic cholinergic receptors with age would be consistent with this finding.

The failure of up-regulation of the nicotinic receptors in smoking schizophrenics was not expected. Approximately $80 \%$ of schizophrenics smoke, compared with 20 $30 \%$ of the general population (Lohr and Flynn 1992; Ziedonis et al. 1994; de Leon et al. 1995), and it has been shown that schizophrenics extract a greater amount of nicotine from each cigarette smoked compared to control subjects (Olincy et al. 1997). While a number of factors should be considered as possible mechanisms for the aberrant regulation of nicotinic receptor levels in schizophrenic brain, the use of neuroleptic medication was a primary concern, as the postmortem tissue used in the present study was primarily from subjects receiving typical neuroleptic medication in life. Therefore, haloperidol was used to assess the effect of chronic neuroleptic and nicotine treatment on nicotinic receptor levels in rat cortex.

Rats treated with the typical neuroleptic haloperidol had the same level of $\left[{ }^{3} \mathrm{H}\right]$-nicotine binding as saline control animals in cortex, indicating that haloperidol alone had no effect on nicotinic receptor expression. Combination treatment with haloperidol and nicotine induced similar increases in nicotine binding as nicotine treatment alone. Further studies have indicated similar results in other rat brain regions (Lee et al. 1998). Interestingly, schizophrenics failed to show any upregulation in thalamus from baseline levels. In thalamus, upregulation in response to nicotine can be reduced and variable, particularly at low doses of nicotine (Marks et al. 1992; Flores et al. 1997; Ulrich et al. 1997; Breese et al. 1997a), indicating that thalamus may be refractory to nicotinic receptor upregulation. While neuroleptic treatment may have affected receptor upregulation of the high affinity receptors in thalamus, the lack of correlation may be related to the expression of other receptor subtypes (Marks et al. 1992) that do not demonstrate the same degree of receptor upregulation to chronic nicotine treatment as the $\alpha 4 \beta 2$ subtype (Fenster et al. 1999).

Although the abnormal regulation of the high affinity neuronal nicotinic receptors in schizophrenia appears to be generally unrelated to chronic neuroleptic treatment, the attenuation of nicotine-induced upregulation in thalamus does suggest possible interactions with other neurotransmitter systems. In addition to the effects of neuroleptic treatment, a recent report suggested that smoking might increase the activity of specific P450 enzymes, leading to increased metabolism of nicotine (Nakajima et al. 1996). However, the experiments reported here do not support such a hypothesis, since haloperidol did not change the up-regulation of receptor levels in rat cortex following chronic nicotine exposure. The haloperidol experiment does not rule out changes due to an interaction of age and long-term neuroleptic treatment, nor effects with atypical neuroleptics such as clozapine.

Previous attempts to measure differences in $\alpha-7$ expression in human postmortem brain using [ $\left.{ }^{125} \mathrm{I}\right]-$ $\alpha$-bungarotoxin homogenate binding have thus far been unsuccessful, primarily due to the extremely low level of expression and high levels of background binding seen with the use of this ligand. In the present study [ $\left.{ }^{3} \mathrm{H}\right]-\mathrm{MLA}$, a potent and selective antagonist at the $\alpha$-BTX binding site (Ward et al. 1990; Davies et al. 1999), was used to measure the $\alpha-7$ receptor levels in membranes from hippocampus and cortex. A small reduction in $\left[{ }^{3} \mathrm{H}\right]-M L A$ binding was seen in schizophrenic subjects in both brain regions, but these differences were not statistically significant. Previous results have shown a reduction in schizophrenic postmortem hippocampus using $\left[{ }^{125} \mathrm{I}\right]-\alpha$-BTX receptor autoradiography (Freedman et al. 1995). Due to the extremely low levels and small numbers of $\alpha-7$ expressing cells in these regions of human brain (Breese et al. 1997b), receptor autoradiography may be a more appropriate method to measure this receptor subtype.

It has been shown in rodents that increases in nicotinic receptor number following chronic nicotine treatment were not due to increases in mRNA levels (Marks et al. 1992). Whether this is true in human brain is currently under investigation. Results from rodent and cell culture experiments suggest that nicotine-induced increases in nicotinic receptor levels result from a modification to the receptor protein as a result of nicotine binding. It has been hypothesized that increases in nicotinic receptor number and the decreased rate of receptor turnover (Peng et al. 1994) are related to the conformational 
state of the nicotinic receptor and subsequent receptor desensitization (Vibat et al. 1995; Baenziger and Chew 1997). A recent report further suggests that receptor desensitization is required for receptor upregulation, particularly the $\alpha 4 \beta 2$ receptor subtype (Fenster et al. 1999).

To fully explain the present results in schizophrenics, the basis of the paradoxical increase in the neuronal nicotinic receptors in normal human smokers must be known. While it could be hypothesized that the nicotinic receptors are not desensitized in schizophrenics and therefore fail to upregulate, data showing a transient effect of nicotine on the normalization of the abnormal auditory gating response in schizophrenics do not support this hypothesis (Adler et al. 1992, 1993). Nevertheless, schizophrenics could have an abnormality in any of several potential mechanisms that change the desensitization state of the receptor and receptor turnover, as well as activation and ion flux through the receptor.

Whatever the mechanism for the reduced levels of both high and low affinity nicotinic receptors in schizophrenics, the resultant reduced nicotinic cholinergic modulation may induce several secondary effects. There is strong evidence that a large component of nicotinic receptor function involves presynaptic receptor activation (Sershen et al. 1995; Wonnacott 1997; MacDermott et al. 1999), and as such would be expected to play an important role as a modulator of numerous neurotransmitter systems. The nicotine-stimulated release of multiple types of neurotransmitters is well-documented (Grady et al. 1994; Rowell 1995; Summers and Giacobini 1995; Bertolino et al. 1997; Liang and Vizi 1997; Wonnacott 1997; Summers et al. 1997; Takahashi et al. 1998). In addition, nicotinic autoreceptors are thought to function at motor-nerve terminals (Bowman et al. 1988; Tian et al. 1994), where they regulate acetylcholine release. This may occur in the brain as well (Rowell and Winkler 1984; Araujo et al. 1988; Wilkie et al. 1996; Summers et al. 1997; Wonnacott 1997).

Presynaptic receptors in brain also appear to regulate the release of GABA (Yang et al. 1996; Alkondon et al. 1997; Wonnacott 1997). A possible regulatory relationship between different nicotinic receptor classes involving inhibition of GABA release in hippocampus has been suggested (Alkondon et al. 1997; Albuquerque et al. 1998). It is interesting to speculate that the decreases in both low and high affinity nicotinic receptor binding seen in schizophrenia might reflect altered receptor subunit ratios in brain, resulting in aberrant inhibitory modulation.

Considering the widespread localization of the neuronal nicotinic receptors and their potential role in modulating numerous neurotransmitter systems in both the brain and periphery, characterization of the nicotinic receptor gene family in schizophrenia and its relationship to the inhibitory deficits and pharmacological medications used in this disease may further the investigation of synaptic modulation in human brain and provide new avenues for treatment.

\section{ACKNOWLEDGMENTS}

This work was supported by USPHS Grants AA11164 and a NARSAD Young Investigator Award to C.R.B., USPHS Grants DA09457 and DA12281 and the VA Medical Research Service to S.L., and USPHS Grants DA03194 and DA00197 to A.C.C.

\section{REFERENCES}

Adler LE, Hoffer LD, Griffith J, Waldo MC, Freedman R (1992): Normalization by nicotine of deficient auditory sensory gating in the relatives of schizophrenics. Biol Psychiatry 32:607-616

Adler LE, Hoffer LD, Wiser A, Freedman R (1993): Normalization of auditory physiology by cigarette smoking in schizophrenic patients. Am J Psychiatry 150:1856-1861

Adler LE, Olincy A, Waldo M, Harris JG, Griffith J, Stevens K, Flach K, Nagamoto H, Bickford P, Leonard S, Freedman R (1998): Schizophrenia, sensory gating, and nicotinic receptors. Schizophr Bull 24:189-202

Albuquerque EX, Pereira EFR, Braga MFM (1998): A basal $\alpha 4 \beta 2$ nicotinic receptor ( $\mathrm{AAChR}$ ) activity controls the release of GABA from hippocampal neurons. Soc Neurosci Abstr 24:1581

Alkondon M, Pereira EFR, Barbosa CT, Albuquerque EX (1997): Neuronal nicotinic acetylcholine receptor activation modulates gamma-aminobutyric acid release from CA1 neurons of rat hippocampal slices. J Pharm Exp Ther 283:1396-1411

Araujo DM, Lapchak PA, Collier B, Quirion R (1988): Characterization of $\mathrm{N}-\left[{ }^{3} \mathrm{H}\right]$ methylcarbamylcholine binding sites and effect of N-methylcarbamylcholine on acetylcholine release in rat brain. J Neurochem 51:292-299

Baenziger JE, Chew JP (1997): Desensitization of the nicotinic acetylcholine receptor mainly involves a structural change in solvent-accessible regions of the polypeptide backbone. Biochemistry 36:3617-3624

Benwell ME, Balfour DJ, Anderson JM (1988): Evidence that tobacco smoking increases the density of $(-)-\left[{ }^{3} \mathrm{H}\right]$ nicotine binding sites in human brain. J Neurochem 50: 1243-1247

Bertolino M, Kellar KJ, Vicini S, Gillis RA (1997): Nicotinic receptor mediates spontaneous GABA release in the rat dorsal motor nucleus of the vagus. Neuroscience 79:671-681

Blaha CD, Allen LF, Das S, Inglis WL, Latimer MP, Vincent SR, Winn P (1996): Modulation of dopamine efflux in the nucleus accumbens after cholinergic stimulation of the ventral tegmental area in intact, pedunculopontine tegmental nucleusles-lesioned, and laterodorsal tegmental nucleus-lesioned rats. J Neurosci 16:714-722

Bowman WC, Marshall IG, Gibb AJ, Harborne AJ (1988): Feedback control of transmitter release at the neuromuscular junction. Trends Pharmacol Sci 9:16-20

Breese CR, Marks MJ, Logel J, Adams CE, Sullivan B, Collins AC, Leonard S (1997a): Effect of smoking history on $\left[{ }^{3} \mathrm{H}\right]$ nicotine binding in human postmortem brain. J Pharmacol Exp Ther 282:7-13

Breese CR, Adams C, Logel J, Drebing C, Rollins Y, Barnhart M, Sullivan B, Demasters BK, Freedman R, Leonard S (1997b): Comparison of the regional expression of nicotinic acetylcholine receptor alpha7 mRNA and [1251]- 
alpha-bungarotoxin binding in human postmortem brain. J Comp Neurol 387:385-398

Court JA, Lloyd S, Thomas N, Piggott MA, Marshall EF, Morris CM, Lamb H, Perry RH, Johnson M, Perry EK (1998): Dopamine and nicotinic receptor binding and the levels of dopamine and homovanillic acid in human brain related to tobacco use. Neuroscience 87:63-78

Dalack GW, Healy DJ, Meador-Woodruff JH (1998): Nicotine dependence in schizophrenia: Clinical phenomena and laboratory findings. Am J Psychiatry 155:1490-1501

Davies AR, Hardick DJ, Blagbrough IS, Potter BV, Wolstenholme AJ, Wonnacott S (1999): Characterization of the binding of $[3 \mathrm{H}]$ methyllycaconitine: A new radioligand for labelling alpha 7-type neuronal nicotinic acetylcholine receptors. Neuropharmacology 38:679-690

de Leon J, Dadvand M, Canuso C, White AO, Stanilia JK, Simpson GM (1995): Schizophrenia and smoking: An epidemiological survey in a state hospital. Am J Psychiatry 152:453-455

Decina P, Caracci G, Sandik R, Berman W, Mukherjee S, Scapicchio P (1990): Cigarette smoking and neurolepticinduced parkinsonism. Biol Psychiatry 28:502-508

Eilers H, Schaeffer E, Bickler PE, Forsayeth JR (1997): Functional deactivation of the major neuronal nicotinic receptor caused by nicotine and a protein kinase C-dependent mechanism. Mol Pharmacol 52:1105-1112

Fenster CP, Whitworth TL, Sheffield EB, Quick MW, Lester RA (1999): Upregulation of surface alpha4beta2 nicotinic receptors is initiated by receptor desensitization after chronic exposure to nicotine. J Neurosci 19:4804-4814

Flores CM, Davila-Garcia MI, Ulrich YM, Kellar KJ (1997): Differential regulation of neuronal nicotinic receptor binding sites following chronic nicotine administration. J Neurochem 69:2216-2219

Freedman R, Coon H, Myles-Worsley M, Orr-Urtreger A, Olincy A, Davis A, Polymeropoulos M, Holik J, Hopkins J, Hoff M, Rosenthal J, Waldo MC, Reimherr F, Wender P, Yaw J, Young DA, Breese CR, Adams C, Patterson D, Adler LE, Kruglyak L, Leonard S, Byerley W (1997): Linkage of a neurophysiological deficit in schizophrenia to a chromosome 15 locus. Proc Natl Acad Sci USA 94:587-592

Freedman R, Hall M, Adler LE, Leonard S (1995): Evidence in postmortem brain tissue for decreased numbers of hippocampal nicotinic receptors in schizophrenia. Biol Psychiatry 38:22-33

George TP, Sernyak MJ, Ziedonis DM, Woods SW (1995): Effects of clozapine on smoking in chronic schizophrenic outpatients. J Clin Psychiatry 56:344-346

Goff DC, Henderson DC, Amico E (1992): Cigarette smoking in schizophrenia: Relationship to psychopathology and medication side effects. Am J Psychiatry 149:1189-1194

Goldstein G, Allen DN, van Kammen DP (1998): Individual differences in cognitive decline in schizophrenia. Am J Psychiatry 155:1117-1118

Grady SR, Marks MJ, Collins AC (1994): Desensitization of nicotine-stimulated $\left[{ }^{3} \mathrm{H}\right]$ dopamine release from mouse striatal synaptosomes. J Neurochem 62:1390-1398

Grenhoff J, Svensson TH (1988): Selective stimulation of limbic dopamine activity by nicotine. Acta Physiol Scand 133:595-596
Heinrichs RW, Zakzanis KK (1998): Neurocognitive deficit in schizophrenia: A quantitative review of the evidence. Neuropsychology 12:426-445

Houghtling RA, Davila-Garcia ML, Kellar KJ (1995): Characterization of $(+/-)(-)\left[{ }^{3} \mathrm{H}\right]$ epibatidine binding to nicotinic cholinergic receptors in rat and human brain. Mol Pharmacol 48:280-287

Hsu YN, Edwards SC, Wecker L (1997): Nicotine enhances the cyclic AMP-dependent protein kinase-mediated phosphorylation of $\alpha 4$ subunits of neuronal nicotinic receptors. J Neurochem 69:2427-2431

Hyde TM, Nawroz S, Goldberg TE, Bigelow LB, Strong D, Ostrem JL, Weinberger DR, Kleinman JE (1994): Is there cognitive decline in schizophrenia? A cross-sectional study. Br J Psychiatry 164:494-500

Jann MW, Saklad SR, Ereshefsky L, Richards AL, Harrington CA, Davis CM (1986): Effects of smoking on haloperidol and reduced haloperidol plasma concentrations and haloperidol clearance. Psychopharmacology 90:468-470

Kaufmann CA, Suarez B, Malaspina D, Pepple J, Svrakic D, Markel PD, Meyer J, Zambuto CT, Schmitt K, Matise TC, Harkavy FJ, Hampe C, Lee H, Shore D, Wynne D, Faraone SV, Tsuang MT, Cloninger CR (1998): NIMH Genetics Initiative Millenium Schizophrenia Consortium: Linkage analysis of African-American pedigrees. Am J Med Genet 81:282-289

Keppel G (1991): Design and Analysis: A Researcher's Handbook, 3rd ed. Englewood Cliffs, NJ, Prentice-Hall, Inc

Lee MJ, Breese CR, Leonard S (1998): The effect of nicotine and haloperidol on high affinity nicotinic receptors and dopamine D2 receptors in the rat brain. J Neurosci 24:833

Leonard S, Adams C, Breese CR, Adler LE, Bickford P, Byerley W, Coon H, Griffith JM, Miller C, Myles-Worsley M, Nagamoto HT, Rollins Y, Stevens KE, Waldo M, Freedman R (1996): Nicotinic receptor function in schizophrenia. Schizophr Bull 22:431-445

Leonard S, Adler LE, Olincy A, Breese CR, Gault J, Ross RG, Lee M, Cawthra E, Nagamoto HT, Freedman R (1998a): The role of nicotine and nicotinic receptors in psychopathology. In Arneric SP, Brioni JD (eds), Neuronal Nicotinic Receptor: Pharmacology and Therapeutic Opportunities. New York, Wiley-Liss, Inc., pp 307-321

Leonard S, Gault J, Moore T, Hopkins J, Robinson M, Olincy A, Adler LE, Cloninger CR, Kaufmann CA, Tsuang MT, Faraone SV, Malaspina D, Svrakic DM, Freedman R (1998b): Further investigation of a chromosome 15 locus in schizophrenia: Analysis of affected sibpairs from the NIMH Genetics Initiative. Am J Med Genet 81:308-312

Leonard S, Logel J, Luthman D, Casanova M, Kirch D, Freedman R (1993): Biological stability of mRNA isolated from human postmortem brain collections. Biol Psychiatry 33:456-466

Liang SD, Vizi ES (1997): Positive feedback modulation of acetylcholine release from isolated rat superior cervical ganglion. J Pharmacol Exp Ther 280:650-655

Lohr JB, Flynn K (1992): Smoking and schizophrenia. Schizophr Res 8:93-102

Luntz-Leybman V, Bickford PC, Freedman R (1992): Cholinergic gating of response to auditory stimuli in rat hippocampus. Brain Res 587:130-136

MacDermott AB, Role LW, Siegelbaum SA (1999): Presynap- 
tic ionotropic receptors and the control of transmitter release. Annu Rev Neurosci 22:443-485

Marks MJ, Collins AC (1982): Characterization of nicotine binding in mouse brain and comparison with the binding of $\alpha$-bungarotoxin and quinuclidinyl benzilate. Mol Pharmacol 22:554-564

Marks MJ, Stitzel JA, Collins AC (1985): Time course study of the effects of chronic nicotine infusion on drug response and brain receptors. J Pharmacol Exp Ther 235:619-628

Marks MJ, Pauly JR, Gross SD, Deneris ES, Hermans-Borgmeyer I, Heinemann SF, Collins AC (1992): Nicotine binding and nicotinic receptor subunit RNA after chronic nicotine treatment. J Neurosci 12:2765-2784

Marks MJ, Smith KW, Collins AC (1998): Differential agonist inhibition identifies multiple epibatidine binding sites in mouse brain. J Pharmacol Exp Ther 285:377-386

McEvoy JP, Freudenreich O, Levin ED, Rose JE (1995): Haloperidol increases smoking in patients with schizophrenia. Psychopharmacology 119:124-126

Miller DD, Kelly MW, Perry PJ, Coryell WH (1990): The influence of cigarette smoking on haloperidol pharmacokinetics. Biol Psychiatry 28:529-531

Nagamoto HT, Adler LE, Hea RA, Griffith JM, McRae KA, Freedman R (1996): Gating of auditory P50 in schizophrenics: Unique effects of clozapine. Biol Psychiatry 40:181-188

Nakajima M, Yamamoto T, Nunoya K, Yokoi T, Nagashima K, Inoue K, Funae Y, Shimada N, Kamataki T, Kuroiwa Y (1996): Role of human cytochrome P4502A6 in C-oxidation of nicotine. Drug Metab Dispos 24:1212-1217

Olincy A, Young DA, Freedman R (1997): Increased levels of the nicotine metabolite cotinine in schizophrenic smokers compared to other smokers. Biol Psychiatry 42:1-5

Olincy A, Ross RG, Young DA, Roath M. Freedman R (1998): Improvement in smooth pursuit eye movements after cigarette smoking in schizophrenic patients. Neuropsychopharmacology 18:175-185

Peng X, Gerzanich V, Anand R, Whiting PJ, Lindstrom J (1994): Nicotine-induced increase in neuronal nicotinic receptors results from a decrease in the rate of receptor turnover. Mol Pharmacol 46:523-530

Pidoplichko VI, DeBiasi M, Williams JT, Dani JA (1997): Nicotine activates and desensitizes midbrain dopamine neurons. Nature 390:401-404

Rapier C, Lunt GG, Wonnacott S (1990): Nicotinic modulation of $\left[{ }^{3} \mathrm{H}\right]$ dopamine release from striatal synaptosomes: pharmacological characterization. J Neurochem 54:937-945

Riley BP, Makoff AM, Mogudi-Carter M, Jenkins TJ, Williamson R, Collier DA, Murray RM (2000): High markerdensity analyses of the $\alpha 7$-nicotinic cholinergic receptor subunit (CHRNA7) gene region on chromosome 15q13q14 and 5' RACE cloning of fragments specific to CHRNA7 or its partial duplication. Schizophr Res 41:93

Romm E, Lippiello PM, Marks MJ, Collins AC (1990): Purification of L-[3H]nicotine eliminates low affinity binding. Life Sci 46:935-943

Rowell PP (1995): Nanomolar concentrations of nicotine increase the release of $\left[{ }^{3} \mathrm{H}\right]$ dopamine from rat striatal synaptosomes. Neurosci Lett 189:171-175

Rowell PP, Winkler DL (1984): Nicotinic stimulation of $\left[{ }^{3} \mathrm{H}\right]$ acetylcholine release from mouse cerebral cortical synaptosomes. J Neurochem 43:1593-1598

Sandyk R (1993): Cigarette smoking: effects on cognitive functions and drug-induced parkinsonism in chronic schizophrenia. Int J Neurosci 70:193-197

Sershen H, Toth E, Lajtha A, Vizi ES (1995): Nicotine effects on presynaptic receptor interactions. Ann NY Acad Sci 757:238-244

Stevens KE, Freedman R, Collins AC, Hall M, Leonard S, Marks MJ, Rose GM (1996): Genetic correlation of inhibitory gating of hippocampal auditory evoked response and alpha-bungarotoxin-binding nicotinic cholinergic receptors in inbred mouse strains. Brain Res 15:152-162

Stevens KE, Kem WR, Mahnir VM, Freedman R (1998): Selective alpha7-nicotinic agonists normalize inhibition of auditory response in DBA mice. Psychopharmacology 136:320-327

Summers KL, Giacobini E (1995): Effects of local and repeated systemic administration of (-)nicotine on extracellular levels of acetylcholine, norepinephrine, dopamine, and serotonin in rat cortex. Neurochem Res 20:753-759

Summers KL, Kem WR, Giacobini E (1997): Nicotinic agonist modulation of neurotransmitter levels in the rat frontoparietal cortex. Jap J Pharmacol 74:139-146

Takahashi H, Takada Y, Nagai N, Urano T, Takada A (1998): Nicotine increases stress-induced serotonin release by stimulating nicotinic acetylcholine receptor in rat striatum. Synapse 28:212-219

Tian L, Prior C, Dempster J, Marshall IG (1994): Nicotinic antagonist-produced frequency-dependent changes in acetylcholine release from rat motor nerve terminals. J Physiol 476:517-529

Ulrich YM, Hargreaves KM, Flores CM (1997): A comparison of multiple injections versus continuous infusion of nicotine for producing up-regulation of neuronal [3H]-epibatidine binding sites. Neuropharmacology 36:1119-1125

Vibat CR, Lasalde JA, McNamee MG, Ochoa EL (1995): Differential desensitization properties of rat neuronal nicotinic acetylcholine receptor subunit combinations expressed in Xenopus laevis oocytes. Cell Mol Neurobiol 15:411-425

Ward JM, Cockcroft VB, Lunt GG, Smillie FS, Wonnacott S (1990): Methyllycaconitine: A selective probe for neuronal alpha-bungarotoxin binding sites. FEBS Lett 270:45-48

Wilkie GI, Hutson P, Sullivan JP, Wonnacoft S (1996): Pharmacological characterization of a nicotinic autoreceptor in rat hippocampal synaptosomes. Neurochem Res 21:1141-1148

Wonnacott S (1997): Presynaptic nicotinic ACh receptors. Trends Neurosci 20:92-98

Xiao Y, Meyer EL, Thompson JM, Surin A, Wroblewski J, Kellar KJ (1998): Rat $\alpha 3 / \beta 4$ subtype of neuronal nicotinic acetylcholine receptor stably expressed in a transfected cell line: Pharmacology of ligand binding and function. Mol Pharmacol 54:322-333

Yang X, Criswell HE, Breese GR (1996): Nicotine-induced inhibition in medial septum involves activation of presynaptic nicotinic cholinergic receptors on gamma-aminobutyric acid-containing neurons. J Pharmacol Exp Ther 276:482-489

Ziedonis DM, Kosten TR, Glazer WM, Frances RJ (1994): Nicotine dependence and schizophrenia. Hosp Commun Psychiatry 45:204-206 